\title{
Zum Zusammenhang von Authentizität und Charisma im Kontext von Selbstzuschreibung, Aushandlung und Angemessenheit - eine exemplarische diskurs- und gesprächslinguistische Analyse
}

\author{
Pamela Steen (Koblenz)
}

\begin{abstract}
How are the concepts of charisma and authenticity jointly constructed in discourse, linked to each other, weighed against each other, played out - in order to build a social or (social) media identity of politicians and other public figures? This paper discusses these questions from a discourse and conversational linguistic perspective. Therefore, particular attention is paid to the self-attribution of charisma and the discursive negotiation of authenticity in connection with the former German Minister of Defense Karl Theodor zu Guttenberg. As an example, sequences from a talk show will be analyzed, which illustrate that the constructed (or demanded) authenticity can be categorized into three interdependent types: person-related (i), situation-related (ii) and ideal (iii) authenticity. Finally, it is postulated that the negotiation of an appropriate form of authenticity is also a question of power.
\end{abstract}

\section{$1 \quad$ Einleitung}

In der britischen Fernsehserie „Downton Abbey“, die zu Beginn des 20. Jahrhunderts spielt, wird auf dem Familiensitz der adligen Familie Grantham erstmals ein Radiogerät abgespielt. Man hört gemeinsam mit den Bediensteten eine Radioansprache von König George V., anschließend unterhält man sich darüber, wie denn der König im Radio „gewirkt“ habe. Im Folgenden werden die Antworten der verschiedenen Figuren auf die Frage des Hausherrn, was man nun von der Radioansprache halte, wiedergegeben (deutsche Übersetzung):

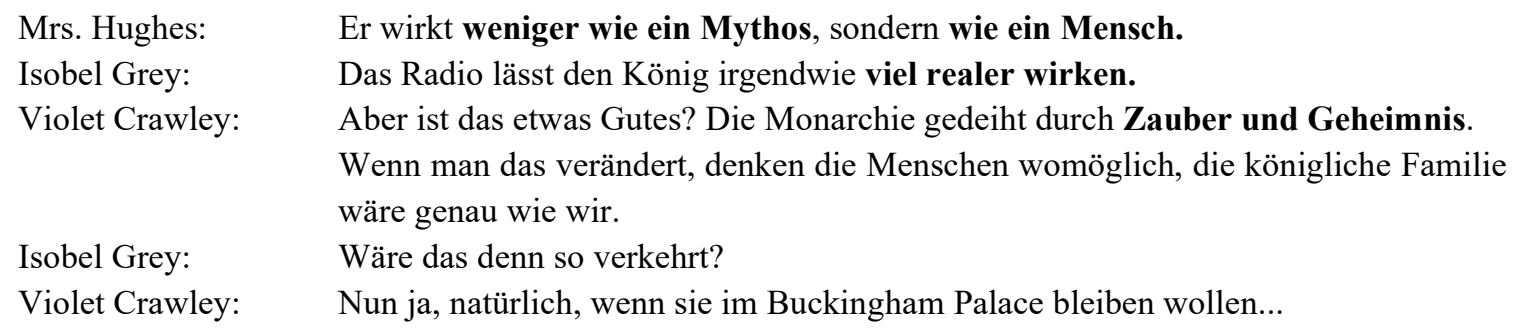

(,Downton Abbey“, Staffel 5 (dt./OV), Folge 2, Episode 36 „Zeitenwende”, Radioszene ab Min. 43:54)

Dass der König durch das Radio medial näher rückt, lässt ihn ,,viel realer wirken“, im Umkehrschluss heißt das (aus der Perspektive der einfachen Angestellten): „weniger wie ein Mythos“. Der König wird zu einem echten Menschen, und damit wird er in einem ganz grundlegenden Sinne authentischer, nicht als Adliger, aber als Mensch. Das hier im fiktionalen Kontext 
verwendete Verb „wirken“ verweist auf die mediale Vermitteltheit der Ansprache. Über das Radio lassen sich die Worte des Königs eindringlicher erfahren, so, als stünde man direkt neben ihm und als säße er nicht auf seinem Thron: „Aus rhetorischer Sicht ist Authentizität als performative Inszenierung von Echtheit, Natürlichkeit, Aufrichtigkeit, Originalität oder Unmittelbarkeit zu begreifen.“ (Ulrich 2012: 79, Hervorhebung i. O.) Die Mittelbarkeit des Mediums und die dadurch inszenierte Authentizität verdeutlichen in dem Beispiel die zuvor unmögliche Unmittelbarkeit der Begegnung mit dem König. Zwar ist der König nach Webers (1976) Einteilung der Herrschaftstypen ein traditionaler Herrscher, aber dass man ihn für etwas Besonderes, etwas Besseres hält, ist nicht allein der Tradition zuzuschreiben, sondern auch dem Glauben an so etwas wie den Mythos des „blauen Blutes“, eben an „Zauber und Geheimnis“. In einer Zeit, in der Adlige ihren Einfluss und ihre Macht immer mehr an demokratisch gewählte Politiker verlieren, ist es wichtig, dass ihr Nimbus, ihr Erb-Charisma, erhalten bleibt. Der König muss auch weiterhin in den Worten Webers die Qualität einer Persönlichkeit besitzen, die als ,außeralltäglich“, als „,magisch bedingt“" gilt,

um derentwillen sie als mit übernatürlichen oder übermenschlichen oder mindestens spezifisch außeralltäglichen, nicht jedem andern zugänglichen Kräften oder Eigenschaften begabt oder als gottgesandt oder als vorbildlich und deshalb als „Führer“ gewertet wird.

(Weber 1976: 142).

Das ist die Perspektive des traditionalistisch eingestellten Familienoberhauptes Violet Crawley: Denn verliert der König aufgrund einer zu gut inszenierten oder auch ungewollt inszenierten Authentizität sein Charisma, dann vielleicht mit ihm alle Adligen und somit auch sie selbst und ihre Familie. Sie hat Angst vor der Veralltäglichung des Charismas, denn der Eindruck einer unmittelbaren räumlichen Nähe bewirkt das Gefühl einer sozialen Nähe, die den konstruierten Nimbus schwinden lässt.

Diese plausible Darstellung eines Gesprächs über Authentizität und Charisma in der fiktionalen „Living History“ - auch wenn beide „catchwords“ des 20. Jahrhundert (Knaller/Müller 2006: 7) nicht verwendet werden - gibt Denkanstöße für eine analytische Auseinandersetzung mit dem heutigen Zusammenhang der beiden Konzepte: Stehen sie in einer Welt, in der der Kontrast von Realität und medial vermittelter Realität verblasst ist, in der real und ,hyper-real“ (Baudrillard 1978) das gleiche zu sein scheinen, noch immer in einem Widerspruch zueinander, oder bedingt das eine gar das andere? Heute ginge es Politikern nicht darum, Authentizitätseffekte zu vermeiden, sondern darum, sie gerade so zu inszenieren, dass ein „bestimmtes Mischungsverhältnis zwischen Privatheit und Öffentlichkeit, zwischen Intimität und Distanz" erzeugt wird, konstatieren Knaller/Müller (2006: 7; cf. auch Thornborrow/van Leeuwen 2001). Der gewählte Politiker soll sich, anders als der traditionelle Monarch, möglichst volksnah geben. Aber wird Volksnähe auch als charismatisch wahrgenommen? Und wie gesellschaftlich legitimiert ist die Selbstzuschreibung von Authentizität und/oder Charisma und welche Auswirkungen auf den Diskurs hat diese?

Um diese Fragen aus einer pragmalinguistischen Perspektive zu beantworten, wird im Folgenden die Methode der Diskursanalyse mit der Gesprächsanalyse verbunden. Die Diskursanalyse gibt einen ersten allgemeinen Überblick über typische Zusammenhänge der beiden Konzepte. 
Es werden Diskursaussagen über Authentizität und Charisma ${ }^{1}$ hinsichtlich unterschiedlicher Sinnebenen systematisiert. Im zweiten Teil erfolgt eine Gesprächsanalyse von Ausschnitten der TV-Talkshow „Menschen bei Maischberger“ (2011), in der die Gesprächspartner/innen über den ehemaligen Verteidigungsminister Karl-Theodor zu Guttenberg diskutieren und dessen mediale Authentizität sowie sein Charisma sprachlich aushandeln. ${ }^{2}$ Die Zusammenführung der beiden pragmatischen Ebenen Diskurs und Gespräch zeigt, dass es stark von den situativen und kontextuellen Umständen abhängt, welche Art von Authentizität, die ein wichtiges Element der rituellen Ordnung darstellt, als angemessen erscheint und wie Art und Angemessenheit sprachlich ausgehandelt werden.

\section{Diskursanalyse: Systematischer Vergleich von Authentizität und Charisma}

Die linguistische Diskursanalyse (cf. Spitzmüller/Warnke 2011) basiert im Folgenden auf zwei Teil-Korpora: Zum einen wurde für die Untersuchung von Charisma ein Korpus (I) aus ca. 3.000 Online-Nachrichtenartikeln aus dem Zeitraum 2008-2014 erhoben. Es erfolgten hierfür auf neun Nachrichtenportalen (TAZ.de, Süddeutsche Zeitung.de, Welt.de, Zeit.de, Handelsblatt.de, Frankfurter Rundschau.de, Frankfurter Allgemeine Zeitung.de, Bild.de Tagesspiegel.de) Suchabfragen mit dem Ausdruck „Charisma“ und morphologisch verwandten Varianten (z. B. „,charismatisch“, „Charismas“, „Charismatiker“) sowie mit Ausdrücken, die häufig im soziologischen Diskurs über Charisma verwendet werden und nach einer ersten induktiven Sichtung des Korpus als in einer semantischen Verbindung mit Charisma stehend verifiziert werden konnten (z. B. „Aura“, „Lichtgestalt“, „Heilsbringer“). Vorrangige Textsorten im Korpus sind Berichte, Reportagen und Kommentare. Um den Umfang des Korpus für eine qualitative Analyse zu begrenzen, wurden nur solche Artikel gespeichert, die sich (im weiteren Sinne) mit deutscher Politik und/oder mit dem damaligen US-Präsidenten Barack Obama beschäftigen. Zum anderen wurde aus diesem Charisma-Korpus (I) ein Teil-Korpus (II) mit Hilfe der Korpus-Analyse-Software MAX QDA gebildet, indem die Ausdrücke „Authentizität“/,,authentisch“, ebenfalls mit Derivationen, gesucht wurden. Dieses Korpus (II) besteht aus 373 Dokumenten. Es handelt sich bei Korpus I und II also um eine die analytische Zugänglichkeit erleichternde systematische Stichprobe, nicht um ein Korpus, das repräsentativ für den ,gesamten“ Diskurs um Charisma in diesem Zeitraum wäre. Zudem ist es ein „Authentizitäts-Korpus“ innerhalb eines Charisma-relevanten Korpus, sodass Zusammenhänge zwischen beiden Konzepten in der einen oder anderen Form anzunehmen sind. Es handelt sich methodologisch betrachtet um Okkurrenztexte, insofern die zu untersuchenden Konzepte durch die Verwendung der genannten Ausdrücke in den Artikeln oberflächennah konstruiert werden. Es wird damit z. B. Felders (2013) Grundpostulat gefolgt, dass linguistische Diskursanalysen an Text- und Gesprächsoberflächen ansetzen, ,um auf dieser Grundlage Perspektivierungen mit Hilfe von divergenten und konvergenten Konzeptualisierungen offenzulegen." (Felder 2013: 20). Gleichwohl lässt sich das Korpus (I, II), bezüglich des Nur-Vorkommens der Ausdrücke, der weiteren Thematisierung von Charisma/Authentizität und ihrer jeweiligen sprachlich-medialen

\footnotetext{
${ }^{1}$ Hier wie im Folgenden ist mit der Kursivsetzung nicht wie sonst in Linguistik online metasprachlicher (objektsprachlicher) Gebrauch, sondern Diskurskonzepte bzw. Begriffe gemeint.

2 Zum Zusammenhang von Pragmalinguistik und Authentizität cf. Deppermann (2000).
} 
Konstruktion auch gegenstandsbezogen in „Okkurrenztexte“, „thematisierende“ und „metasprachlich-reflexive“" Texte aufteilen. ${ }^{3}$

Bereits bei erster Durchsicht der Texte fällt auf: Für beide Begriffe gilt, was Knaller/Müller (2005: 40) für Authentizität formuliert haben, dass es keine eindeutige Definition, weder in historischer, noch in aktueller Perspektive geben könne. $\mathrm{Zu}$ heterogen sind die Bereiche, in denen der Ausdruck verwendet wird, und die etymologische Entwicklung eröffnet ein differentes semantisches Feld (cf. Knaller/Müller 2005: 40; Dietschi 2012; Ulrich 2012). Insofern können auch die hier angeführten Diskursbelege, die den Bereich Politik in einem bestimmten zeitlichen Abschnitt betreffen, nur typische Zusammenhänge von Authentizität und Charisma erkennbar machen, jedoch nicht allumfassend definieren. Es geht zunächst darum, für die spätere Gesprächsanalyse zu einem konkreten Fall von Authentizität in Verbindung mit Charisma einen allgemeinen diskursiven Hintergrund zu skizzieren.

Beide Wörter sind typischerweise Hochwertwörter, mit denen Politikern personale Eigenschaften zu- oder abgeschrieben werden:

Zwar sagt man für gewöhnlich, eine Person habe Charisma, es bedeutet jedoch dann viel eher, dass einer Person eine charismatische Ausstrahlung von einer Interpretationsgemeinschaft zugeschrieben wird.

(Jentges 2010: 77; Hervorhebung i. O.)

Der interpretative Authentizitätsbegriff schließlich versteht A. nicht als falsifizierbare Eigenschaft oder anzustrebendes Ideal (,,authentisch sein“), sondern als Resultat eines Zuschreibungsprozesses (,,authentisch erscheinen").

(Ulrich 2012: 80; Hervorhebung i. O.)

Authentizität und Charisma werden auch im Zuge der folgenden Diskursanalyse in methodologischer Hinsicht als interpretative Begriffe verstanden, deren Bedeutung von verschiedenen Faktoren im Diskurs abhängig ist. ${ }^{4}$ Es ist das Ziel der exemplarischen Analysen, einige Faktoren aufzuspüren und sichtbar zu machen. Aus den Artikeln im Korpus wurden hierfür insgesamt sechs Sinnebenen ermittelt, die die Begriffe systematisierend zugänglicher machen. Diese Ebenen sind: „Merkmale“ (1), „Akteure“ (2), „Zuschreibung“ (3), „System“ (4), „Diskursereignis“ (5), „Zeit“ (6). Diese Sinnebenen geben Antworten auf die Fragen: „Was ist C./A.?“ (1); und mit welchem Akteur B wird der charismatische/authentische Akteur A verglichen?“ (2); ,wer schreibt wem C. und A. zu/ab?“ (3); „welche Rolle spielt das politische System bei der Zu-/Abschreibung?“ (4); „mit welchem Diskursereignis wird C./A. in Verbindung gebracht?“ (5); ,auf welche historische Phase wird C./A. bezogen konstruiert?““(6).

\subsection{Merkmale: Was charismatisiert/authentifiziert (nicht)?}

Charisma wird im Korpus zumeist als ein Bündel von Eigenschaften/Merkmalen konstruiert, die dem Politiker (als Charismaträger) zugeschrieben werden. Es werden Argumente für und

\footnotetext{
${ }^{3} \mathrm{Zu}$ dieser Unterscheidung cf. auch Steen (2015a).

${ }^{4}$ Es wurde bereits an anderer Stelle der Zusammenhang zwischen dem Konzept Charisma und seinem Entstehen in Kommunikationsprozessen sowie die Analyse von Charisma in Verbindung mit der Methode der linguistischen Diskursanalyse dargelegt: Siehe hierzu Steen (2015a) sowie Steen (2017).
} 
gegen Charisma vorgebracht. Es wird also danach gefragt: Was charismatisiert oder was charismatisiert nicht? Charisma wird dabei oft über die Negation bestimmt:

[1] „Charisma ist kein Tugendpreis, Charisma setzt sich nicht dem auf den Schoß, der am anständigsten ist. Vielleicht gehört ja auch die Vermessenheit zum Charisma und die Maßlosigkeit, wie sie einst Franz Josef Strauß eigen war.“

(Prantl 2010a, Süddeutsche Zeitung)

Charisma wird in dieser Diskursaussage eines thematisierenden Textes nur scheinbar definiert. Die Metaphern „Tugendpreis“ und ,auf den Schoß setzen“ lassen ebenso wie das Adverb „vielleicht" Vagheit in der Bedeutung entstehen. Die Referenz auf Strauß mit den beiden pejorativen Substantiven „Vermessenheit“" und „Maßlosigkeit“" vermitteln den Eindruck, dass Charismatisierungen gerade denjenigen Politikern zu Teil werden, die sie zumindest von einer moralischen Seite her nicht verdient hätten. Gleichzeitig wird mit dem Besitz-Verb „gehören“ nur gesagt, dass diese Eigenschaften Teile von Charisma sein können, das Gesamtkonzept wird damit jedoch nicht definiert. Auch in den folgenden Belegen aus Okkurrenztexten wird nicht eindeutig definiert, was Charisma ist, stattdessen wird aufgezählt, welche Eigenschaften die Politiker/innen stattdessen besitzen, die aber nicht hinreichend oder gar notwendig für Charisma sind:

[2] „Hannelore Kraft hat kein Charisma, und dieser Mangel wird nicht durch Spritzigkeit oder Gewitztheit ausgeglichen.“

(Schloemann 2010, Süddeutsche Zeitung)

[3] „Wulff mag persönlich nett und voller guter Absichten sein, er bleibt ein Mann ohne Charisma."

(Fücks 2012, Süddeutsche Zeitung)

„Spritzigkeit“, „Gewitztheit“ [2], ,persönlich nett“", ,voller guter Absichten“ [3] - das sind personale Eigenschaften, die einen Politiker nicht unverwechselbar machen, sondern lediglich als einen normalen Menschen erscheinen lassen. Es wird dabei jedoch nicht gesagt, dass Charismatiker nicht nett oder spritzig sein dürften, nur ist dies eben keine hinreichende Bedingung für eine Charismatisierung, die für beide Politiker/innen aber notwendig sein soll, um politisch erfolgreich zu sein.

Häufig lassen Aufzählungen von Eigenschaften - wie diejenige im folgenden Beleg - die Leser/innen darüber im Unklaren, ob sich diese Eigenschaften ergänzen oder aber zueinander in einer Teil-Ganzes-Relation stehen (cf. auch Steen 2017):

[4] „Obama habe ,unheimliches Charisma“, sei ,sehr eloquent‘ und ,einer der intelligentesten Menschen in unserem Land“".

(Wolff 2010, Süddeutsche Zeitung)

Wenn Eloquenz und Intelligenz zum Charisma als notwendige Eigenschaften gehören [4], so ist die Aufzählung zumindest teilweise redundant und „Charisma“ lediglich ein catchword. Wenn sich die Eigenschaften ergänzen, so wirkt der Ausdruck „Charisma“, zumal mit dem Intensivierer „unheimlich“, mystifizierend, aber nicht erklärend, da nichts über diese Qualität ausgesagt wird. Zudem steht er dann gleichberechtigt neben den beiden anderen (allerdings ebenfalls durch Intensivierer und Superlative gekennzeichneten) Eigenschaften und wird dadurch zu einem Merkmal unter anderen. 
Im Falle von Authentizität sind die Konstruktionen ähnlich. In der folgenden Belegstelle wird gesagt, was ,authentisch sein“ nicht ist:

[5] „Ansonsten finden sich Pressemitteilungen, Grußbotschaften und Bilder, auf denen Merkel hier mal jenem die Hand schüttelt und dort mal dem anderen. Authentisch geht anders."

(Denkler 2015, Süddeutsche Zeitung)

Indem Merkel macht, was alle Politiker ständig machen: Hände schütteln, sich volksnah geben [5] - sich nach den Routinen der Politik als Politikerin inszenieren -, erfüllt sie gerade nicht die Vorstellung von einem authentischen Handeln, vor allem, weil dieses (in „Grußbotschaften und Bildern") medial auf Facebook inszeniert wird. In einer anderen Aussage wird deutlich, dass das, was im Gegenzug ungewöhnlich, unerwartet ist, authentisch wirkt:

[6] „Ansgar Belke, Professor für Volkswirtschaft in Essen sagt: ,Wir waren großzügig genug durch Fiskaltransfers und Troika', aber das lässt Steinbrück so nicht stehen. ,Noch viel mehr unserer Solidarität könnte möglich sein.' Hier wirkt Steinbrück authentisch. Das ist keine populäre Position.“

(Eichhorn 2013, Bild)

Authentizität wird Steinbrück zugeschrieben, weil dieser von dem Handeln, das man normalerweise von ihm oder anderen Politikern erwartet hätte, abweicht [6]. Die Erwartung bezieht sich darauf, dass Steinbrück normalerweise eine populäre und möglicherweise für sein Image auch weniger gefährliche Position vertreten hätte. Stattdessen sagt Steinbrück etwas, das unerwartet ist. Dass es Steinbrücks Strategie gewesen sein könnte, auch entgegen seiner persönlichen Meinung, an diesem Tag „ein unbequemer Gesprächspartner“ zu sein oder besonders zu „emotionalisieren" wird nicht erwogen. Es ist lediglich das offensichtliche Differenzverhalten, das authentisch macht. Und dieses hängt von der jeweiligen subjektiven Perspektive des Zuschreibenden und dessen Verhaltenserwartungen ab. Diese Perspektive stützt sich auf bereits generalisierte Erwartungen. Denn es ist immer das erwartete typische Handeln von Politikern, das nicht authentisch sein kann. Anders ausgedrückt: Der Politiker/die Politikerin, der/die macht, was andere nicht erwarten, wird als authentisch wahrgenommen. Dahinter steht eine bestimmte Deontik, sich anders geben zu müssen als die anderen oder als die Erwartungen der anderen an das eigene Handeln vorgeben. Hier eröffnet sich jedoch eine paradoxe reflexive Spirale, die authentisches spontanes Handeln beinahe unmöglich macht. Denn es kommt nicht auf ein ,authentisches authentisches“ Handeln an, sondern nur auf ein ,authentisch inszeniertes authentisches Handeln“, sodass gerade Politiker ,ihre Authentizitätsinszenierungen häufig professionell einüben“" (Knaller/Müller 2006: 8).

Während das Konzept Charisma eher mit durativen personalen Eigenschaften im Allgemeinen konzipiert wird, die jemand haben kann oder auch nicht (wie Eloquenz, Intelligenz, Vermessenheit), wenngleich diese oft Performanzqualitäten beschreiben, wird das Konzept Authentizität eher mit einer transitorischen Performanz konzeptualisiert. Man ist authentisch/nicht authentisch in einem singulären Augenblick, der verschiedene Handlungsmöglichkeiten bietet, an deren Auswahl man sich messen lassen muss. Durativ wird Authentizität z. B. aber dann, wenn mit dem Ausdruck auf im Diskurs bereits als charismatisch identifizierte Politiker referiert wird: 
[7] „Es ist nun nicht zuletzt diese Art des zaudernden Regierens, das bei nicht wenigen Wählern und Journalisten eine Sehnsucht nach vermeintlich authentischen Charakteren produziert, nach der leidenschaftlichen Symbolik eines Willy Brandt, den mitreißenden Reden eines Herbert Wehner, ja selbst nach der narzisstischen Emphase eines Helmut Kohl.“

(Markwardt 2015, Zeit)

Hier wird Merkel, die ,zaudernd“ regiert, Authentizität in einer generellen Weise abgeschrieben [7]. Wenngleich den Politikern Brandt, Wehner und Kohl Authentizität mit eingeschränkter Validität (,vermeintlich“) zuerkannt wird, wird deutlich, dass deren Performanz nicht einfach nur beschrieben, sondern charismatisierend beschrieben wird mittels des Adjektivs ,leidenschaftlich“, des Partizips „mitreißend“ sowie des Substantivs „Emphase“. Diese Lexeme lassen die Personen als hochgradig emotional bzw. emotionalisierend erscheinen. Bei Brandt und Wehner sind es keine Lexeme, die die Emotion, also einen individuellen inneren Vorgang der Politiker explizit und direkt benennen (cf. Schwarz-Friesel 2007: 144; Hermanns 1995), wie individuelle Angst, Freude oder Zorn, sondern Brandt verwendet eine Symbolik, die leidenschaftlich ist. „Symbolik“ ist als metasprachliches Zeichen ein Zeichen für ein zeichenhaftes Konstrukt, das auf etwas anderes verweist. Ist diese Symbolik leidenschaftlich, so ist es der Verweis eines Verweises auf Emotion. Brandt selbst muss jedoch nicht emotional gewesen sein. Wehner führte Reden, die andere in einen emotionalen Zustand des „Mitgerissenseins“ versetzten. Er ist Auslöser von Emotionen anderer, über die aber explizit nichts gesagt wird, außer, dass sie sich positiv von seinen Reden affizieren ließen. Auch Kohls „Emphase“ beschreibt nicht direkt einen inneren Zustand, denn Emphase ist ein emotionaler Performanzausdruck. Man kann nicht an sich emphatisch sein, sondern die Emphase ist ablesbar an einer Handlung, die jemand mit Emphase, also eindringlich, ausdrucksstark, energisch, glühend usw. vorführt. Das Adjektiv ,narzisstisch“ beschreibt diese Emphase bei Kohl außerdem als ein reflexives Phänomen, als ein Sich-an-sich-selbst-Berauschen. Die Lexeme referieren also nur generalisierend und indirekt auf emotionale Zustände. Wenn diese Politiker auch nicht selbst als emotionale Menschen beschrieben werden, so bewirken sie aber indirekt eine emotionale Aura, die durch Dynamik, nicht durch Stillstand oder eben „Zaudern“ gekennzeichnet ist und die ein interpretatives, kein empirisches Konstrukt ist. Authentisch erscheinen diese drei Politiker, weil sie in einzelnen Situationen der Performanz - anders als die breite Masse der Politiker - immer wieder emotional oder emotionalisierend wirken, sodass ein ,authentischer Charakter“ entsteht. Authentische Emotionalität führt zur Charismatisierung. Und obwohl diese bei Politikern nicht die Norm ist, wird sie normativ gesetzt, denn Merkel wird an diesen Ausnahmen gemessen.

Es zeigt sich, was Knaller/Müller (2006: 8) über den schillernden Begriff Authentizität schreiben, nämlich, dass dieser häufig ,,auf nicht immer aufschlüsselbare Weise empirische, interpretative, evaluative und normative Elemente miteinander verknüpft". Dies gilt ebenso für den Charisma-Begriff, wobei es allerdings so scheint, dass es letztlich so viele Charisma-Arten wie Charismatiker gibt, sich „Authentizität“ jedoch nicht als individueller, sondern als ein bestimmter typischer Performanzausdruck darstellt. Jeder ist auf seine eigene Weise charismatisch, das ist eine notwendige Bedingung für Charisma. Helmut Kohl kann nicht das Charisma eines Willy Brandt haben und vice versa. Charisma ist damit eher eine positive Performanzfigur, während Authentizität eine negative Differenzfigur ist, die nur in einem ,etwas anders machen 
als es in der politischen Routine erwartbar wäre" interpretierbar wird. Diese wahrgenommene Differenz kann dann jedoch ein Argument für Charismatisierung sein.

\subsection{Akteure: Welches Beziehungsgeflecht konstruiert Charisma/Authentizität?}

Wenn Charisma personengebunden ist, kann es, wie schon das Beispiel ,Merkel im Kontrast zu Kohl und Co. ‘ [7] zeigte, im Diskurs als Differenzgröße verwendet werden, um Politiker voneinander zu unterscheiden.

[8] „Beim ,deutschen Obama“ schwingt mit, was der CSU derzeit fehlt: jugendliche Dynamik gepaart mit einer Extraportion Charisma.“

(Kruse/Das Gupta 2010, Süddeutsche Zeitung)

[9] „Er mag kein großer Charakterkopf sein, vom Charisma eines Guttenberg ist er Lichtjahre entfernt.“

(Kruse/Jakat 2011, Süddeutsche Zeitung)

[10] „In Amerika sind Charismatiker jung und glatt und schön. In Deutschland sind sie schon älter, sie sind angefressen vom Zahn der Zeit, und sie kriegen beim Reden einen roten Kopf. Aber darauf kommt es nicht an, es kommt darauf an, wie einer redet und wie er die Leute packt."

(Prantl 2010, Süddeutsche Zeitung)

Über das Konzept Charisma werden drei Vergleichsgrößen eröffnet [8]: Obama, Guttenberg und die anderen Politiker der CSU. Zunächst wird Guttenberg mit Obama verglichen, verdichtet in der Konstruktion „deutscher Obama“, die eine semantische Blend (cf. Fauconnier 1997) aus Obama und Guttenberg entstehen lässt. Somit geht das Charisma Obamas mittels dieser Blend auf den deutschen Politiker über. Explizit wird dies jedoch erst, wenn wiederum Guttenberg mit der „CSU“, also den CSU-Politikern in einem kollektivierenden Ausdruck verglichen wird. So muss sich die gesichtslose Menge der CSU-Politiker mittelbar mit dem US-amerikanischen Präsidenten vergleichen lassen. Charisma wird auch hier wieder als eine personale Eigenschaft konstruiert, die vage bleibt, die aber Politiker dennoch miteinander vergleichbar macht. Dies wird im zweiten Beleg [9] noch deutlicher: Das „Charisma eines Guttenberg“, eigentlich eine individuelle Größe, da sie an den Charismaträger „Guttenberg“ gebunden ist, wird als relationale Größe konstruiert. Den Eindruck von Objektivität bewirkt die Verwendung des indefiniten Artikels („eines Guttenbergs“), die Guttenberg als Typus konstruiert, wodurch auch dessen Charisma typisiert und damit vergleichbar wird. In Beleg [10] wird amerikanisches und deutsches Charisma ebenfalls typisierend miteinander verglichen und eine Differenz konstruiert. Das amerikanische Charisma (Obamas) wird nach ästhetischen Kriterien beurteilt, das deutsche nach Performanz-Kriterien (Lafontaines). Im Amerika-Deutschland-Vergleich wird Lafontaines Charisma gegen das „eindeutige“, weil auf den ersten Blick erkennbare Charisma von Obama, verteidigt: Auch das ist Charisma, wenn einer einen „roten Kopf“ bekommt. Beide Politiker, Obama und Lafontaine, stehen hier zudem für einen Charisma-Typus, der metonymisch auf die gesamte „Charismatiker-Riege“ des jeweiligen Landes übertragen wird, zwei Jahre bevor Guttenberg als Obama-ähnlicher Charismatiker konstruiert wird. Es zeigt Beliebigkeit, vorgebliche Generalisierbarkeit und Objektivierbarkeit, mit der Politikern über das Vergleichsschema (cf. Kienpointner 1992) Charisma zu- und abgeschrieben wird. Dass ein Politiker Charisma hat, ist hier nicht die These, sondern das Argument dafür, einen (anderen) 
Politiker oder eine Politikergruppe abzuwerten. Dies geschieht im Korpus (II) auf ähnliche Weise mit dem Hochwertwort ,Authentizität“"

[11] „Hoeneß ist alles andere als eine Figur, die auf Lug und Trug aufgebaut war [...]. ,Ich schwitze in der Nacht, ich wälze mich. Und denke nach, denke nach und verzweifle', sagt er im Interview mit der ZEIT. So viel Authentizität haben Guttenberg und Wulff nicht hingekriegt."

(Esslinger 2013, Süddeutsche Zeitung)

[12] „Der große Vorteil, den Rösler gegenüber Westerwelle hat: Er ist dabei authentisch und hat den nötigen Schuss Selbstironie.“

(Denkler/Jakat 2011, Süddeutsche Zeitung)

[13] „Dieter Kronzucker, einst Gründer des heute-journal des ZDF und seit vielen Jahren das politische Gesicht des Nachrichtensenders N24, hat eine Lehre aus dem Obama-Wahlkampf gezogen. Damit ein deutscher Politiker ebenso erfolgreich sein kann, egal ob nun im TV oder im Internet, muss er authentisch und bereit sein, auch Persönliches von sich preiszugeben. Dieter Kronzucker hat auch eine Vorstellung, welcher deutsche Politiker diese Anforderung erfüllen könnte: Wirtschaftsminister zu Guttenberg.“

(Sagatz 2009, Tagesspiegel)

Der Fußballmanager Hoeneß wird mit zwei Politikern verglichen, um den Umgang mit seinem Fehlverhalten zu relativieren und damit indirekt auch sein Fehlverhalten in gewisser Weise zu rechtfertigen [11]. Dabei wird Hoeneß' Authentizität, die sich darin zeigt, dass er offen und emotionalisierend über sich selbst spricht, mit der mangelnden Authentizität von Guttenberg und Wulff verglichen. Die reine Aussage des Akteurs über Emotionalität, die selbst keine Performanz von Emotion ist, wird zum Authentizitätsmarker. Denn der Ausdruck „Schwitzen“ bezeichnet nur einen möglichen Indikator für Reue, ist es aber selbst nicht. Und auch ,,verzweifeln“ ist „selber aber gar nicht emotiv und expressiv“, sondern nur ein „Gefühlswort“, eine „quasi-psychologische Vokabel“ (Hermanns 1995: 145-147). Mit einer „distanzierten, deskriptiven Benennung“ (Hermanns 1995: 145) seiner Emotionen kann also jemand schon authentisch wirken. Lediglich die Konstruktionswiederholung ,und denke nach, denke nach“ ist ein performatives Indiz für Emphase, allerdings auch nur die vergangenen Vorgänge illustrierend. Am Beispiel Guttenberg wird später noch genauer untersucht, welche Äußerungen dieser bei der Presseerklärung zur Plagiatsaffäre macht und wie diese Äußerungen interpretiert werden. Hoeneß, das muss betont werden, agiert nicht in der Rolle eines Politikers, sondern Fußballmanagers und ehemaligen Fußballers. Dies ist eine andere soziale Rollen-Rahmung, mit der der Vergleich inadäquat wird. Wenn sich Nationalmannschaftstrainer Rudi Völler in einem Interview (2006), das bei YouTube mit dem Titel „Rudi Völler rastet aus“ eingestellt wurde, emotional zeigt, so heißt es hinterher in den Kommentaren des sozialen Netzwerks: „In erster Linie Mensch, der Rudi.....deshalb mag ich ihn so! Richtig gemacht! Gib ihnen Saures!“ In der Fußballbranche ist authentische, d. h. unkontrolliert wirkende Emotionalität im Gegensatz zur Politik, wo Unkontrolliertes mit Irrationalität gleichgesetzt wird, nicht per se imageschädigend, 
wenn das positive Image bereits hergestellt ist. Politiker, die sich zu emotional, d. h. distanzlos emotional geben, werden dafür eher kritisiert. ${ }^{5}$

In Beleg [12] werden zwei Politiker, analog zu den Charisma-Beispielen [8], [9], miteinander verglichen, wobei sich Westerwelle an der Authentizität Röslers messen lassen muss. In Beleg [13] ist es wieder Guttenberg, der mit Obama verglichen wird, nun hinsichtlich seiner generellen Authentizität (bevor es zur Plagiatsaffäre kam).

Relationalität, so kann zusammengefasst werden, spielt demnach bei der $\mathrm{Zu}$ - und Abschreibung von Charisma und Authentizität jeweils eine wichtige Rolle in der Argumentation pro oder contra Politiker. Sie ist damit Indikator dafür, dass ,,verschiedene Akteure um Aufmerksamkeit [konkurrieren] und [...] dadurch unter bestimmten Handlungszwängen [stehen], die die Chancen einer massenmedialen Inszenierung von Repräsentanten beeinflussen und strukturieren." (Jentges 2010: 85). Kein Politiker ist allein auf der Bühne, er befindet sich automatisch in einem sozialen Geflecht aus Akteuren, die über sprachliche Kategorisierungen positioniert werden. Das soziale Geflecht, das sich im Diskurs widerspiegelt und auch über diesen konstruiert wird, kann mit Elias als „Figuration“ verstanden werden, ,die viele interdependente Individuen miteinander bilden“ (Elias 1997: 15). Figurationen binden einzelne Menschen und ihre Motive aneinander und bringen sie dazu, in einer ganz spezifischen Weise zu handeln, in der sie vielleicht nicht handeln würden, wenn sie frei von diesen Interdependenzen wären (cf. Baumgart/Eichener 1997: 103). Die Belegbeispiele zeigen insofern, dass es auch um die Frage „Wer gegen wen?" geht (cf. Jentges 2010: 51), denn der Politiker als Repräsentant

[...] muss mindestens zwei Handlungszonen erreichen, weil er sowohl in der Welt seiner Konstituenten nach innen als auch in der Welt seiner politischer (sic!) Konkurrenten und potenziellen Partnern nach außen agiert, wobei er nach außen in unzählig viele Kontexte eingebunden sein kann.

(Jentges 2010: 51)

\subsection{Zuschreibung: Wer charismatisiert/authentifiziert wen?}

Ein weiterer Aspekt dieser Figurationen ist die Zuschreibung als solche, d. h. schreibende Akteure (Autor/innen) können in diesen Figurationen, wie die obigen Beispiele zeigen, Charisma/Authentizität zu- oder abschreiben. Sie können aber auch selbst ein Zuschreibungsnetz konstruieren, indem Fremd-Positionierungen (cf. das Positionierungskonzept nach Harré/van Langenhove 1999) über die beiden Hochwertwörter vorgenommen werden. Es kommt dabei zu Perspektivverschiebungen.

[14] „Merkel bewundert (und beneidet) Obama wegen seines Charismas.“

(Kornelius 2010a, Süddeutsche Zeitung)

\footnotetext{
5 Dabei kommt es allerdings auf die Art der Emotion an. Ein gerührter Joe Biden, der weint, als ihn Obama im Januar 2017 verabschiedet, ist eine positive menschliche Ausnahme in der Politik („Emotionale Überraschung am Ende der Amtszeit“", wie der Titel eines YouTube-Video der Welt-Reporterin Lorenz 2018 lautet) und damit authentisch und sympathisch. Wenn Wolfgang Bosbach wutentbrannt die Maischberger-Talkrunde verlässt, heißt es hinterher im Internet (allerdings eher nicht von Seiten der CDU-Anhänger): „Kindischer Abgang, der zu solchen Typen passt“ (NN5 2017, Merkur). Wobei es auch Stimmen gibt, die argwöhnen, der Eklat könnte geplant, Bosbachs Reaktion damit nicht authentisch gewesen sein.
} 
[15] „Steinmeier machte sich anheischig, die internationale soziale Marktwirtschaft, für die Merkel mit Sprödigkeit wirbt, zu einem sozialdemokratischen Projekt zu machen. Er hascht nach Charisma."

(Prantl 2010b, Süddeutsche Zeitung)

Es ist nun Merkel, die Obamas Charisma bewundert und damit auch implizit zuschreibt, wenngleich dieses hier als objektives Datum konstruiert wird [14]. Aber wesentlich an dieser Aussage ist das Gefühlsverb „,beneiden“, das das Fehlen von Merkels Charisma semantisch dadurch ausdrückt, dass man nicht jemanden um etwas beneiden kann, das man selbst hat. So wird sprachlich eine Figuration konstruiert, deren Logik sich auf soziale Rollen und Positionen stützt (beide sind die mächtigsten Politiker/innen in ihrem Land), aber eben auch auf perspektivierte konstruierte persönliche Emotionen. Diese Diskursaussage vereint damit beide gesellschaftliche Seiten: die ,individuellen, psychologischen Strukturen, also [...] [die] sogenannten Persönlichkeitsstrukturen, und [...] [die] Figurationen, [...] [die] Sozialstrukturen“ (Elias 1997: 15). Wenn Steinmeier ,nach Charisma hascht“ [15], dann wird darin die Zuschreibungspraxis selbst implizit thematisiert, es ist damit also eine latent meta-sprachlich-reflexive Aussage, aber zumindest eine metapragmatisch-reflexive, insofern Steinmeier unterstellt wird, er wisse oder hoffe, dass bestimmte Handlungen (die internationale soziale Marktwirtschaft zu einem sozialdemokratischen Projekt zu machen) zu einer Charismatisierung seiner Person durch andere führen könne. Auch in dieser Aussage wird damit die psychologische Struktur zumindest implizit angesprochen, die in die Charismatisierungsprozesse und in die relevanten Figurationen involviert ist.

[16] „Derweil hat sich auch Bundeswirtschaftsminister Karl-Theodor zu Guttenberg zur Kritik an Merkels Wahlkampfstil geäußert. Der CSU-Politiker verteidigte Merkels ,Strategie, die auf Nüchternheit setzt ${ }^{‘}$. Es sei ein großer Vorteil im Wahlkampf, ,dass Angela Merkel authentisch bleibt"“.

( $\mathrm{NN}_{1}$ 2010a, Süddeutsche Zeitung)

[17] „Auch die entwaffnende Art der beiden Erstwähler ließ die Politiker authentischer werden. Und genau das ist es ja, was Wähler sich von ihren Kandidaten wünschen, anstelle ungekonnter Twitter- oder Facebook-Aktivität.“

$\left(\mathrm{NN}_{3} 2013\right.$, Bild $)$

Auch die Zuschreibung von Authentizität wird den politischen Diskursakteuren übertragen. Guttenberg wird mit einer direkten Rede zitiert, wodurch die Zuschreibung explizit als subjektiv erkennbar ist [16]. Gerade im Kontext des Wahlkampfes, der durch Guttenberg sprachlich aufgerufen wird, wird deutlich, dass Zuschreibungen von Politikern selbst einen direkten politischen Nutzen haben sollen, da Guttenberg und Merkel über die beiden Schwesterparteien figurativ verbunden sind. In Beleg [17] wird zunächst ein Einfluss der Erstwähler auf das Verhalten der Politiker postuliert, um anschließend diese explizit als ein Kollektiv fremdzupositionieren, das sich Authentizität von den Kandidaten wünscht. Damit wird die Figuration hier nicht nur durch Perspektiven, Handlungen und Einflüsse konstruiert, sondern auch erstaunlich passend gemacht: Das Kollektiv A (Erstwähler) verhält sich auf eine bestimmte Weise und löst damit beim Kollektiv B (Politiker) eine Art der Reaktion aus, die zufällig von A gewünscht, aber nicht intendiert ist und die darin besteht, ein authentisches Verhalten zu zeigen. Die 
Intentionen des Kollektivs A dürften vielmehr sehr unterschiedlicher Art gewesen sein (z. B. dass B zuhört oder Fragen beantwortet). Damit wird die Art der Reaktion von B als ein „Phänomen der dritten Art" konstruiert, als eine „kausale Konsequenz einer Vielzahl individueller intentionaler Handlungen, die mindestens partiell ähnlichen Intentionen dienen." (Keller 1994: 92; Hervorhebungen i. O.). „Authentischer werden“ die Politiker, weil sie direkt reagieren und nicht indirekt über soziale Medien. Authentizität ist hier also ein steigerungsfähiger Wertungsbegriff (cf. Knaller/Müller 2005: 45). Und auch hier wird, wie unter $2.1 \mathrm{im} \mathrm{Be-}$ leg zu Merkel [5], die Benutzung der (sozialen) Medien als Ursache für inauthentisches Wirken angegeben.

Anders als im fiktionalen Eingangsbeispiel, bei dem der britische König durch Mediennutzung authentischer wirkt, werden die Politiker durch Face-to-Face-Kommunikation wieder authentischer (die aber mit dem König ohnehin nicht möglich ist). Dass die Medien bei dem Treffen der Politiker mit den Erstwählern in der Talkshow von Maybrit Illner dennoch in Form von Kameras vor Ort waren und die Situation beeinflusst haben, wird hier in der Konstruktion von Authentizität ausgeblendet. Entscheidend ist, dass sowohl Merkel als auch die hier angegebenen Politiker darin scheitern, über soziale Medien mit den Wählern eine soziale Nähe zu konstituieren, die man für authentisch halten könnte.

Interessant an der Aussage ist vor allem, dass die Behauptung, die Politiker agierten authentischer, aus der Beobachterperspektive des Dritten vollzogen wird. Wenn nun behauptet wird, das sei es auch, was sich die Wähler wünschten, so wird gleichzeitig postuliert, dass auch diese aus ihrer eigenen Perspektive heraus Authentizität wahrnähmen. Dies ist jedoch eine doppelt ungesicherte Behauptung.

Der Kontrast zwischen authentisch/inauthentisch weist immer auf eine ontologische Differenz zwischen Repräsentant und Repräsentiertem hin, und die Feststellung der Authentizität/Inauthentizität setzt dann voraus, daß der (wissenschaftliche) Beobachter selbst über eine adäquate Sicht auf die richtige Repräsentation verfügt. Dieser privilegierte Wirklichkeitszugang ist aber epistemologisch nicht zu begründen.

(Noetzel 1999: 12)

Somit wird in der obigen Diskursaussage ein zweimaliger privilegierter Wirklichkeitszugang behauptet: 1. Man wüsste, was in dieser Situation authentisch/inauthentisch ist; 2. Man wüsste, dass auch die Wähler „die Wirklichkeit“ in der gleichen Weise sehen oder dass diese Authentizität zumindest auf sie einwirkt. Dass die Zuschreibung ,X ist authentisch/inauthentisch in

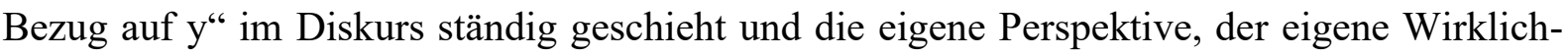
keitszugang, nicht hinterfragt wird, ist jedoch die Regel (und nicht etwa nur schlechter BildJournalismus), weshalb danach gefragt werden muss, welche zeichenvermittelte Realität mittels dieser Behauptungen konstruiert wird. Hier ist es der privilegierte Blick auf eine ideale Politiker-Wähler-Situation, die aufgrund der Authentizität der Politiker, die nur deshalb plausibel ist, weil sie nicht intendiert ist, zwei Generalisierungen über die Wirklichkeit impliziert: 1. Politiker sind im Allgemeinen wenig authentisch und im Besonderen, wenn sie soziale Medien verwenden, und 2. Diese Politiker haben es geschafft, in einer wichtigen Situation authentischer zu werden, weshalb sie sich von der breiten Masse positiv abheben; Daraus folgt 3. Wenn sie authentischer sind, sind sie wählbar, denn das entspricht dem Wunsch aller Wähler. 
Authentizität, so zeigt sich, wird im Diskurs vorrangig als intersubjektiver Begriff verwendet (cf. Ferrara 1998).

\subsection{System: Welche Bedeutung hat Charisma/Authentizität für das politische System?}

An anderer Stelle (cf. Steen 2015a) wurde gezeigt, dass es einen diskursiven Zusammenhang von Charismatisierungen und dem in den Zeitungsartikeln konstruierten politischen System gibt. Die untersuchten Diskursaussagen lassen sich dabei in drei Kategorien einteilen: 1. Charisma ist notwendig für Demokratie. 2. Charisma ist nützlich für Demokratie. 3. Charisma ist unvereinbar mit Demokratie. Es konnte gezeigt werden, dass hinter diesen Bewertungskategorien verschiedene Konzepte von Charisma sowie von Demokratie stehen. So korrelieren Demokratisches Charisma, Postdemokratisches Charisma, Herrschaftscharisma und PseudoCharisma auf unterschiedliche Weise mit Parlamentarischer Demokratie, Postdemokratie und Mediendemokratie. Hierzu das Beispiel einer Headline:

[18] „Charisma macht müde Demokratien munter.“

(Burmester 2014, Berliner Republik)

Wenn Diskursakteure Charisma als ein Mittel betrachten, um Bürger/innen wieder für Politik zu begeistern, „müde Demokratien munter“ zu machen, so ist dies ein postdemokratisches Charisma. Charisma wird zum Heilmittel, um das jetzige politische System zu transzendieren (cf. Steen 2015a, 8). Dabei ist diese Mittel-Zweck-Relation ein häufiges Diskursmuster, wenn Charisma in Beziehung zum politischen System gesetzt wird. Auch Authentizität wird in dieser Form relational zum politischen System konstruiert:

[19] „Die Piraten wollten menschlich bleiben, echt, authentisch, sie wollen die Liquid Democracy schaffen, mehr demokratische Teilhabe organisieren, dem erstarrten Profipolitiker ein menschliches Antlitz geben.“

(Körner 2013, Tagesspiegel)

Dieser Wunsch der Piraten erinnert etwas an eine ,totale demokratische Teilhabe aller Gesellschaftsmitglieder“, um das „Larvenspiel“ der politischen „Maskenträger“ zu beenden, wie Noetzel (1999: 13) dies für den rousseauistischen Authentizitäts-Diskurs zusammenfasst. Authentizität soll auch bei den Piraten zu einer energetischeren, flüssigeren Demokratie führen, die derzeit nur noch aus „erstarrten Profipolitikern“ besteht - ein Hinweis auf die Idee einer postdemokratischen Entwicklung [19]. Es wäre sicherlich lohnenswert, auch für das Konzept Authentizität systematisch im Diskurs zu rekonstruieren, mit welchen Demokratie-Konzepten es korrelierend konstruiert wird. Konnte für das Konzept Charisma gezeigt werden (cf. Steen 2015a), dass die in der Soziologie gebräuchlichen Charisma-Auffassungen im Mediendiskurs parallel existieren und das semantische Feld in Bewegung ist, so ist beispielsweise anzunehmen, dass es im Hinblick auf Authentizität vor allem in Verbindung mit dem Konzept der Mediendemokratie relevante Aussagen gibt, da „die Medien“, wie die obigen Belege zeigen, eine Schlüsselfunktion bei der Konstruktion von Authentizität zu haben scheinen. Schließlich gilt es, herauszufinden, ob sich im Diskurs auch Aussagen finden, die Noetzels Postulat von einer zwingenden Abhängigkeit von Authentizität und der Legitimität der politischen Ordnung widerspiegeln: „Ohne den Anspruch des Authentischen ist weder auf der Ebene der individuellen Folgebereitschaft noch der system-funktionalen Legimitätserzeugung politische Ordnung 
herstellbar. (Noetzel 1999: 16). Das Fehlen von Authentizität oder zumindest die Wahrnehmung einer mangelnden Authentizität von Politikern könnte demnach zu politischen Krisen führen, da es die politische Ordnung gefährdet, während die Vorstellung von einem authentischen Charismatiker zu einem Nonplusultra einer globalen Demokratie-Idee wird:

[20] „Hat dieser Obama die Formel für den guten Populismus, den Weg zu einer neuen Nähe zum Volk und das Rezept für die Restauration des Vertrauens in die Politik gefunden? Ist Obama der erste authentische Hoffnungsträger für die Demokratien in einer globalisierten $\mathrm{Zu}$ kunfts-Weltgesellschaft?““

(Perger 2008, Die Zeit)

\subsection{Diskursereignis: ${ }^{6}$ Welche Ereignisse beeinflussen die Konstruktion von Cha- risma/Authentizität?}

Charisma und Authentizität sind „Krisenbegriffe“. Diese Einschätzung findet sich in der Literatur z. B. bei Sennett und bei Knaller/Müller:

Die große Karriere des Authentizitätsbegriff in der zweiten Hälfte des 20. Jahrhunderts scheint darin begründet zu sein, daß der Einzelne in seiner Rolle als allgemeiner Mensch, als besonderes Mitglied von Gemeinschaften, Organisationen und als unvergleichliches, singuläres Individuum keinen Ort mehr findet, von dem aus er in der „obdachlosen“ Moderne diese unterschiedlichen Zumutungen zu synthetisieren vermöchte. So gesehen ist der Authentizitätsbegriff Ausdruck und zugleich Symptom dieser Krise, also ein Krisenbegriff, der die Krise erfaßt, selbst stets in der Krise ist und zugleich als ,Zauberwort“ die Krise zumindest partiell unsichtbar macht.

(Knaller/Müller 2006: 10f.)

Während der Authentizitätsbegriff die Krise des Individuums in einer gesichtslosen Gesellschaft beschreibt, ist Charisma, zumindest noch bei Weber, Ausdruck der Krise der Gesellschaft. Die Masse verlangt in krisenhaften Situationen nach einem Menschen, den sie ,mit der Aura gottähnlicher Macht“ (Sennett 2004: 345) ausstatten kann. Lenze (2002: 142) schreibt hierzu ganz ähnlich über das postmoderne Charisma: „Für jede sozial-deviante Situation, für jede (auch die kleinste) Krise kann das entsprechende charismatische Objekt gefunden werden, das die bestehende „Notlage“ in ihr dialektisches Gegenüber überführt.“ Auch nach Freud, so Sennett, braucht es den charismatischen Führer, um Krisen der Massen zu verhindern: „Freud zufolge muß es in jeder Gesellschaft charismatische Führer geben, denn ohne sie wären die Massen jederzeit bereit, die Gesellschaft ins Chaos zu stürzen." (Sennett 2004: 346). Doch Sennett, aus seiner kulturkritischen Perspektive der 1970er Jahre, sieht die gesellschaftliche Krise in Verbindung mit Charisma beim charismatischen Individuum selbst angesetzt. Wenn es dem vermeintlichen Charismatiker nur aufgrund seiner telegenen Attraktivität gelingt, Wählerstimmen zu erhalten, wenn ,der Golf spielende oder mit einfachen Leuten zu Tisch sitzende Präsident" (Sennett 2004: 349) die Menschen davon ablenkt, ,sich über die unerfreulichen Seiten des Lebens Gedanken zu machen“ (Sennett 2004: 350), so ist Charisma nicht mehr wie bei Freud und Weber die „Reaktion auf gesellschaftliche Unordnung“ (Sennett 2004: 350;

\footnotetext{
${ }^{6}$ Diskursereignisse werden hier verstanden als einerseits selbst von den Medien konstruiert, bei denen andererseits aber auch „ein Diskurs besonders stark in Erscheinung tritt“ (Link 1983, cf. Spitzmüller/Warnke 2011: 108). Hier sind es Ereignisse, die die Verwendung der Ausdrücke „Authentizität“ und „Charisma“ wahrscheinlicher machen, vor allem politische Wahlen und Krisen.
} 
Hervorhebung i. O), sondern dann ist ,das moderne Charisma [...] die Ordnung selbst und produziert gerade als solche Krisen.“ (Sennett 2004: 350). Aus soziologischer Sicht bilden (post)modernes Charisma und Krise demnach ein polyvalentes Beziehungspaar. Die Belegstellen im Korpus zeigen ebenso einen Zusammenhang, der mindestens auf drei Arten konstruiert wird:

1) Das Charismatische als Mittel zur Lösung:

[21] „Amerika will einen Neuanfang. Mit zwei Kriegen und einer Finanzkrise belastet, sehnt sich das Land nach einer Befreiung. An der Urne hat Amerika seine Handlungsfreiheit zurückgewonnen. Mit ihrer Stimme stemmte sich die Mehrheit der Wähler gegen den Niedergang, den Bedeutungsverlust und gegen das politische Missmanagement, das den Abstieg nur noch beschleunigte. Barack Obama hat es am besten verstanden, der Sehnsucht des Landes Ausdruck zu verleihen. Wer eine Mobilisierungs-Kampagne dieser Schlagkraft organisiert, wer Charisma und Zuversicht verströmt, wer selbst unter größtem Druck Gelassenheit zeigt und Distanz wahrt, der wird auch ein guter Präsident sein.“

(Kornelius 2010b, Süddeutsche Zeitung)

2) Charisma als Ursache der Krise:

[22] „Doch die Partei steckte im Frühjahr 2011 in einer veritablen Krise. Ihre Lichtgestalt KarlTheodor zu Gutenberg hatte gerade wegen einer gefälschten Doktorarbeit zurücktreten müssen und Parteichef Horst Seehofer geriet in Not.“

(Hebestreit 2013, Frankfurter Rundschau)

3) Das Uncharismatische als Mittel zur Lösung:

[23] „Seriös, bieder bürokratisch, zuverlässig: Das schlägt glamourös, charismatisch, forsch, unterhaltsam. Das Gefühl der Dauerkrise, die keiner durchschaut, gebiert offenbar eine neue Sehnsucht.“

(Lehming 2012, Tagesspiegel)

Dass Obama selbst hierzulande zu Beginn und bereits vor seiner Karriere als US-Präsident als eine charismatische Ausnahmeerscheinung wahrgenommen wurde [21] $]^{7}$, ist offenbar wesentlich seiner Rhetorik zu verdanken, der Weibler (2010: 20) eine Konstitution von „Unmittelbarkeit“ bescheinigt. Zudem sei dabei ,die Authentizität des Gesehenen und Gehörten in sich stimmig und mit der Botschaft vereinbar" (Weibler 2010: 21):

Wer in Krisenzeiten durchtrainiert wirkt und bei aller gebotenen Seriosität locker und stressfrei daherkommt, jung aussieht und für die Aufgabe tatsächlich vergleichsweise jung ist und verständlich spricht, dabei noch an einen anderen jungen und beliebten Präsidenten im Amte erinnert, ohne ihn zu kopieren, der besitzt diese Authentizität und zwar solange, bis das Gegenteil bewiesen ist oder sich von Misserfolg auf hinter der dann nur vermuteten Maske liegende Brüche und Abgründe vermeintlich schließen lässt.

(Weibler 2010: 21f.)

\footnotetext{
7 „Dabei sitzt der Demokrat erst seit zwei Jahren im Senat. Was ihm an Kompetenz fehlt, macht er durch Charisma wett“ ( $\mathrm{NN}_{2}$ 2010b, Süddeutsche Zeitung); „Wenn am Donnerstag Zehntausende unter der Siegessäule in Berlin Obama nahe sein wollen, dann geht es nicht um das Kleingedruckte im transatlantischen Wahlprogramm. Sie wollen das Charisma spüren, die Kraft der Worte und die Bedeutung von Politik“ (Kornelius 2010c, Süddeutsche Zeitung).
} 
Authentizität, so könnte zusammengefasst werden, ist gerade in Krisenzeiten ein Mittel, um ein Charisma zu konstruieren/zu postulieren, nach dem sich viele sehnen, um die Krise zu bewältigen. Was bei Weibler eher positiv bewertet wird, klingt bei Sennett wiederum kulturpessimistisch. Auch er fragt danach, wie es einem Politiker gelingt, als charismatisch wahrgenommen zu werden. Es ginge darum, von der politischen Überzeugung, die nicht jedermann teilt, abzulenken und die eigene Motivation deutlich zu machen. Dies geschieht, indem der Politiker die Aufmerksamkeit auf seine inneren Regungen lenkt und damit zu einem glaubwürdigen Führer wird,

indem er den Anschein erweckt, er handele gemäß seinen inneren Impulsen spontan und bewahre gleichzeitig seine Selbstkontrolle. Gelingt ihm diese kontrollierte Spontaneität, so erscheinen seine Regungen wirklich, und dann kann man ihm glauben.

(Sennett 2004: 343)

„Vor diesem Hintergrund kann der Politiker als aktiver Mann erscheinen, auch wenn er in seinem Amt gar nichts leistet.“ (Sennett 2004: 343). Dass Guttenberg für charismatisch und authentisch gehalten wurde, was sich dann in der Plagiatsaffäre ins Gegenteil verkehrte, führte zu einer Diskursaussage wie [22]. Dass ausgerechnet die „Lichtgestalt" Guttenberg zurücktreten musste, stürzte die Partei in eine ,veritable Krise“. Guttenberg wurde letztlich vorgeworfen, was Sennett für alle charismatisierten Politiker annimmt, dass dieser nicht authentisch, sondern immer schon unglaubwürdig gewesen sei (siehe dazu die Gesprächsanalyse unten). Eigenschaften wie ,glamourös, charismatisch, forsch, unterhaltsam“ [23] will dann niemand mehr haben, weil es scheint, dass diese persönlichen Eigenschaften „,das Gefühl der Dauerkrise“ nicht beenden. Aber erst aus der Kontrasterfahrung mit Guttenberg, mit der impliziten Kontextualisierung der Plagiatsaffäre wird diese Diskursaussage verstehbar. Denn erst die Enttäuschung mit dem Charismatiker führt zur Verherrlichung des „Biederen“. Das Biedere kann dabei auch das Authentische sein:

[24] „Wäre es nicht schwer abgenutzt, müsste man sagen, sie kommt so authentisch und glaubwürdig daher, dass ihr Kind und Hund folgen. Offenbar sucht das Publikum in Krisenzeiten verschärft nach dem Unverstellten, dem zuverlässigen Ort, um Vertrauen zu parken.“

(Mika 2012, Frankfurter Rundschau)

Während es im Korpus, wenig überraschend, keine Fundstellen gibt, die das Inauthentische in Krisenzeiten als Sehnsuchtsobjekt konstruieren, Authentizität aber generell als ein Begriff verwendet wird, ,der insofern an etwas Krisenhaftes gebunden ist, als er situiert ist in problematischen Situationen von Unsicherheit und Unwissenheit, in denen durch Authentizität eine Evidenz zu schaffen ist, die es anders nicht gibt“ und er somit auch ein „epistemologischer Begriff“ ist (Krämer 2012: 25f.), ist die Konstruktion des Charisma-Begriffs stärker von gesellschaftlichen Entwicklungen und Einzelereignissen abhängig. Er ist zwar ebenso ein Krisen-Begriff, aber mehr in einem differenzierten Sinne, insofern Charisma als Ursache, Lösung oder Verschlechterung von Krisen angesehen wird, je nachdem, um welche Art Krise es sich zu welchem Zeitpunkt handelt.

\subsection{Zeit: Welche epochale Bedeutung wird Charisma/Authentizität zugeschrieben?}

Obwohl Charisma der Begriff ist, der stärker an die Entwicklung von Einzelereignissen gebunden zu sein scheint als Authentizität, werden im Korpus beide als Sehnsuchtsbegriffe an 
bestimmte Zeitabschnitte oder Epochen gebunden. Dieser größere, allgemein zeitliche Zusammenhang wird deutlicher, wenn die Sehnsucht sich auf etwas richtet, das dauerhaft nicht wiederzuerlangen ist. Denn für die Postmoderne gilt, dass beide Begriffe, ganz im Sinne von Sennett, nur noch auf etwas abzielen, das mit der erfolgreichen Vermarktung des Selbst zu tun hat: So heißt es bei Knaller/Müller: „Authentizität mit seiner (sic!) Aura von Echtheit, Wahrhaftigkeit, Ursprünglichkeit, Unmittelbarkeit, Eigentlichkeit ist zu einem erfolgreich eingesetzten Markenartikel und Emblem geworden (Knaller/Müller 2006: 7). Ganz ähnlich klingt die Beschreibung von Charisma bei Lenze:

Handelt es sich beim magischen Charisma jedoch um ,echte' Magie, so ist das postmoderne ,Pseudo-Charisma‘ durch ,Magie 2. Grades‘ konstituiert: Durch Magie also, die alltäglichem rationalen Handeln einen scheinbar tiefen Sinn verleihen will. Nichts anderes produzieren beispielsweise die unzähligen Werbeagenturen in ihrem alltäglichen Eifer, immer wirkungsvollere, emotionale Markenwelten hervorzubringen, um im Alltag, Inseln von Außeralltäglichkeit‘ zu schaffen.

(Lenze 2002: 131)

So müssen also „echte“ Authentizität und „echtes“ Charisma heute etwas Unerreichbares sein. Postulate bezüglich einer neuen Ära werden auch in einigen Diskursaussagen implizit deutlich: [25] „Wehner, das war klar, würde man im Parlament immer zuhören. Das war sein genuiner Politikort. Authentisches Leben spielte sich hier ab, Politik pur nämlich. Beides war eins. Plus Butterstulle, die ihm die Stieftochter regelmäßig brachte zum Durchhalten.“

(Hofmann 2009, Die Zeit)

Authentizität wird hier nicht einer Person, sondern einer ganzen politischen Epoche zugeschrieben, in der es noch „Politik pur“ gab. Da war das „Leben“ selbst authentisch, insofern es um nichts anderes ging als um Politik. Heute, im „Internetzeitalter“, ist das politische Leben weitgehend von Inszenierungen in den sozialen Medien beeinflusst:

[26] „Die Sehnsucht nach einer politischen Erlöserfigur und einem Messias, dessen Botschaft über den Tag hinaus reicht, der mit anderen, mit authentischen Zungen spricht und der die aussöhnenden Gesten beherrscht, ist groß. Regiert werden wir dennoch von Madame Effizienz, Angela Merkel, deren kühle Aura deshalb so gut zum Internetzeitalter passt, weil all die Augenblicksalarme, die situativ geborenen Dramen, all die Entblößungs- und Durchleuchtungsschauspiele des digitalen Marktplatzes an ihr abperlen. Kann man jemanden entzaubern, dem kein Zauber innewohnt?“،

(Körner 2013, Tagesspiegel)

Als Kontrast zu Obama als einer authentischen charismatischen Sehnsuchtsfigur wird Merkels Identität als jenseits von Authentizität und Charisma konstruiert. Das „Internetzeitalter“ besitzt Entzauberungspotenzial (so wie etwa bei Guttenberg, der über die Webseite „GuttenPlag“ entzaubert wurde), selbst für diejenigen Charismatiker, die „mit authentischen Zungen“ sprechen, weil die Gefahr der Entlarvung der Magie als Magie 2. Grades immer gegeben ist. Während Wehner also für ein politisches Zeitalter steht, das an sich authentisch war [25], stehen Obama und Merkel jeweils auf ihre Weise für das inauthentische Internet-Zeitalter [26]: Der eine bedient es, so lange er kann, es sich nicht gegen ihn wendet und wirkt dabei authentisch und 
charismatisch; die andere entzieht sich ihm und damit auch jeglicher emotionalisierender, charismatisierender Zuschreibung.

Diese diskurslinguistischen Beispielanalysen vermitteln einen Eindruck davon, welche Bedeutungen die Konzepte Authentizität und Charisma im Diskurs haben. Im Wesentlichen wurde gezeigt, dass die Ausdrücke „Charisma“ und „Authentizität“ nicht einfach Hochwertwörter sind, die die Identitäten von Politikern positiv beschreiben, sondern dass sie in vielfältigen Sinnzusammenhängen weitere Bedeutungsfacetten erhalten. So entfalten die Begriffe, die mit ihren jeweiligen Merkmals-Schwerpunkten als Performanz- und Differenzfiguren beschrieben wurden, in Abhängigkeit von konstruierten sozialen Figurationen, politischen Systemen, Diskursereignissen und allgemeinen Epochenkonstruktionen jeweils ihre interpretative, relationale, intersubjektive und normative Facette.

\section{Gesprächsbeispiel: Karl Theodor zu Guttenberg - Charismatiker mit Authentizi- tätsproblem}

Am Beispiel von Guttenberg, der Plagiatsaffäre und dem öffentlichen Umgang mit Guttenbergs offizieller Entschuldigung wird nun der Zusammenhang von Diskursaspekten und dynamischer Aushandlung von Charisma und Authentizität im Gespräch fokussiert.

Auf dem Höhepunkt seiner Karriere 2009 gibt Guttenberg in einer Interview-Reihe von meinVZ.net ein Interview und spricht darin von Charisma und Authentizität:

[27] „Also, es kann sein, dass ich völlig uncharismatisch bin, das müssen natürlich andere für sich dann letztlich beurteilen. Und in meiner Beurteilung dann auch anlegen. Aber ich versuche eines, nämlich authentisch zu bleiben. Das ist das Entscheidende. Und mich nicht nur den Fliehkräften derer zu unterwerfen, die versuchen, einen irgendwo einzunorden. Und das ist mein Rezept. Ob's gelingt, ist 'ne andere Frage."

(Guttenberg 2009)

In seiner Rede zeigt Guttenberg, dass er reflektiert, dass er auf einer Bühne steht. Indem er über die Zuschreibungsebene aufruft, dass andere beurteilen müssen, ob er charismatisch sei, kokettiert er damit, dass er weiß, dass dies andere bereits tun. Im Akt der Reflexion von Authentizität - mit dem Kopula-Verb „,bleiben“ spricht er sich zudem implizit in diesem Moment zu, authentisch zu sein - beweist er nach Knaller/Müller aber das Gegenteil: „Wendet man sich reflexiv auf diesen Akt des Zeigens zurück, ist man aus der Authentizität herausgefallen und redet - auf nicht authentische Weise - über Authentizität.” (Knaller/Müller 2006: 9). Und bei Sennett heißt es dazu ähnlich:

Je mehr sich eine Person auf die Authentizität ihres Fühlens statt auf den objektiven Gehalt dessen, was sie fühlt, konzentriert, je mehr Subjektivität zum Selbstzweck wird, desto weniger vermag sie, expressiv zu sein. (...) Der Satz ,Schau, wie ich fühle‘ ist ganz offensichtlich von Narzißmus geprägt.

(Sennett 2004: 49).

Es ist die deskriptive Meta-Ebene, nicht die performative Ebene, die Guttenberg hier in Bezug auf Authentizität ansteuert. Interessanterweise überlässt er anderen die Beurteilung, ob er charismatisch sei, die Beurteilung, ob er authentisch sei, will er jedoch nicht als intersubjektive, sondern als subjektive Kategorie verstanden wissen und dabei die Deutungshoheit über seine 
Person behalten. Er setzt Authentizität zudem mit Autonomie im Handeln gleich. Dass er sich jedoch Gedanken über die „Fliehkräfte“ macht und sein Verhalten in Differenz zu diesen ausrichtet, zeigt bereits, dass er nicht mehr autonom agiert. Eigentlich müsste Guttenberg angesichts derartiger Aussagen weder als charismatisch, noch als authentisch wahrgenommen werden, wenn es stimmt, was Jentges über die Notwendigkeit des Repräsentanten schreibt, nämlich, dass dieser, „um den politischen Körper authentisch“ auszufüllen, diesen „aus tiefstem Inneren empfinden [muss]. Er muss sozusagen vergessen, dass er auf der politischen Bühne schauspielert" (Jentges 2010: 63). Aufgrund seiner adeligen Herkunft hatte Guttenberg jedoch viele Anhänger:

[28] „Es gibt ein Bedürfnis nach einer authentischen Biografie, die sich vom üblichen abhebt, beobachtet Thomas Steg. Extremstes Beispiel ist der adlige Verteidigungsminister zu Guttenberg $[\ldots]^{\text {“ }}$

(Hildebrandt 2010, Die Zeit)

Guttenberg wurde von vielen als authentisch und charismatisch („vom üblichen abhebend“) wahrgenommen, wie es der ehemalige Regierungssprecher Steg zusammenfasst. Ende 2010 erklärt der Soziologe Hartmann in einem Zeitungsinterview, wie Guttenbergs Erfolg zustande kommt:

[29] „Immer, wenn das Annähern an den abwesenden Minister kurz vor der Heiligsprechung angelangt ist, streut Professor Michael Hartmann von der Technischen Universität Darmstadt Sand ein. Er [...] hat beobachtet, dass Guttenbergs Erfolg vor 20, 30 Jahren nicht möglich gewesen wäre. Das politische Personal habe mittlerweile ein derart geringes Ansehen, dass es zu einem solchen Aufstieg kommen könne [...]. Die Leute würden vom gelobten Minister gute Entscheidungen erwarten, da er den Job eigentlich nicht nötig habe. Er besitze ja genug Geld und könne sich sozusagen Authentizität leisten."

(Jakobs 2010, Süddeutsche Zeitung)

In Hartmanns Erklärung wird die Zeitebene angesprochen: Er vergleicht den heutigen Politikbetrieb mit dem ,vor 20, 30 Jahren“ und kommt bezüglich der Zuschreibung sowie der anderen beteiligten Akteure zu dem Schluss, dass die Bürger Politiker heute allgemein geringschätzen. Guttenberg kann jedoch authentisch sein, weil er ,genug Geld“ hat und deshalb autonom agieren kann. Damit wird der Adel zum Konstituens von Charisma und Authentizität, weil dieser ihm nicht nur Unabhängigkeit, sondern eben auch eine „Biografie“ beschert, die sich vom üblichen abhebt“" [28]. Weil Guttenberg adlig ist, ist er finanziell unabhängig, kann er sagen, was er wirklich denkt und fühlt, wirkt er charismatisch. Anders als im fiktionalen Eingangsbeispiel gefährdet hier Authentizität nicht das Charisma des Adligen, sondern bewirkt es. Denn der Adlige hebt sich hier in der Differenzfigur nicht von anderen Adligen ab, die dem Volk nicht sozial näher kommen, sondern von anderen, ,,normalsterblichen“ Politikern, die es gar nicht schaffen können, authentisch zu sein.

Mit der Plagiatsaffäre verschob sich die Charisma-Authentizitäts-Balance. Guttenberg hatte Teile seiner Doktorarbeit gefälscht, plagiiert, und nun war er nicht mehr glaubwürdig. Die mangelnde Glaubwürdigkeit war Resultat des Skandals, der wiederum aus der Enthüllung entstand. Hondrich (2002: 14-17) macht in der Entwicklung eines politischen Skandals typischerweise vier Schritte aus: den Fehltritt (Guttenbergs Plagiat), die Enthüllung (organisiert durch 
„Guttenplag“), die Entrüstung (z. B. öffentlich in TV-Talkshows) und die Genugtuung (das von Guttenberg geforderte Schuldeingeständnis, der Rücktritt als Verteidigungsminister).

Guttenbergs Glaubwürdigkeitsproblem wurde mit Authentizität und Charisma in Beziehung gesetzt. In der Talkshow „Menschen bei Maischberger“" vom 22.02.2011 mit dem Titel „Der Schummelbaron - Frechheit siegt?“ kann ein dynamischer Aushandlungs-Prozess im Sinne einer kollektiven Entrüstung beobachtet werden. Maischbergers [Mai] Gäste sind: Prof. Dr. Arnulf Baring [Bar] (Historiker), Jutta Ditfurth [Dit] (Gründungsmitglied der Grünen), Prinzessin Anna von Bayern [Bay] (Guttenberg-Biografin, Bild am Sonntag), Dr. Werner Schneider [Sch] (österreichischer Kabarettist), Norbert Geis [Gei] (Parteifreund von Guttenberg), Ulrike Demmer [Dem] (Der Spiegel). Sequenzen der Talkshow (mit Dialoganteil oder simultanem Sprechen) wurden mit dem Partitureditor EXMARaLDA nach den Transkriptionskonventionen des Minimaltranskripts/GAT 2 (cf. Selting et al. 2009) transkribiert.

Die Entrüstung, schreibt Hondrich (2002: 15), „folgt der Enthüllung schlafwandlerisch“. Erfolgt die kollektive Entrüstung, ist der Skandal da. Es wird im Folgenden rekonstruiert, mit welchen sprachlich-rhetorischen Strategien die Akteure in ihrer „Entrüstungsrunde“ Authentizitätskriterien aufrufen und verhandeln, welche Widersprüche sich dabei zeigen und welche sozialen Funktionen die Authentizitätskonstruktionen haben (cf. hierzu auch Deppermann 2000, siehe für eine soziolinguistische Auseinandersetzung mit Authentizität z. B. Lacoste/Leimgruber/Breyer 2014).

\subsection{Sprachliche Charismatisierung und De-Charismatisierung}

Obwohl, oder gerade weil es um das Fehlverhalten von Guttenberg geht, wird dieser in der Sendung weiterhin sprachlich charismatisiert. Die Charismatisierung erfolgt durch Anna von Bayern, die seine Biografie geschrieben hat, sowie durch Norbert Geis, einen Parteifreund:

\begin{tabular}{|lcccc|}
\multicolumn{1}{c}{} & \multicolumn{1}{c}{80} & 81 & 82 & 83 \\
\hline Sch $[\mathrm{vb}]$ & \multicolumn{2}{c}{ hoffentlich } & HOFFentlich & familie nicht \\
Gei $[\mathrm{vb}]$ & allein & sond ern seine ganze familie $(-)$ seine ganze familie er geht durch
\end{tabular}

[135]

\begin{tabular}{|c|c|}
\hline & 84 \\
\hline Dit [vb] & ein sta:hlgewitter (-) jetzt sind wir wieder beim krieg \\
\hline Gei $[\mathrm{vb}]$ & ein sta:hlgewitter durchund da geb ich ihnen recht herr baring es ist \\
\hline
\end{tabular}

Geis vergleicht die Situation, in der sich Guttenberg befindet, mit einem „sta:hlgewitter“, durch das er und „seine ganze familie“ (82-83) jetzt durchgehen. Damit wird ein persönliches Krisenereignis konstruiert, das die Art seines Auftretens in den Medien entschuldigen soll. Mit der Kontextualisierung des Buches von Ernst Jünger In Stahlgewittern wird Guttenberg zudem heroisiert. Die Plagiatsaffäre wird überhöht und Guttenberg selbst implizit als Opfer dargestellt. Ditfurth wiederholt den Ausdruck (84) und kritisiert ihn als unangemessen, indem sie ihn explizit in den Kriegskontext stellt. Schneider macht die Heroisierung an anderer Stelle (nicht im 
Transkript) metapragmatisch explizit: „das hat so etwas Heldenhaftes.“ Es ist hier der Versuch von Geis, Guttenbergs zuvor im Diskurs konstruiertes Charisma zu konservieren, indem die Plagiatsaffäre mit persönlicher Bedeutung aufgeladen wird, während die anderen Akteure versuchen, Guttenberg zu de-charismatisieren, indem sie ihn über die Betonung, es sei ein gewöhnliches Tricksen gewesen, zu entzaubern versuchen. Figurationssoziologisch wird hier deutlich, dass der „Held immer ein Held einer Interpretationsgemeinschaft ist, die in Größe, Zusammensatzung und Dauer sehr verschieden sein kann“" (Jentges 2010: 71). In dieser Gesprächsrunde ist sie extrem fragil, bzw. Geis gelingt es nicht (mit Ausnahme von Anna von Bayern), die anderen Akteure von seiner Interpretation der Geschehnisse zu überzeugen. Aus anthropologischer Perspektive zeigt sich dennoch, was Lipp für Heldenkonstruktionen annimmt: Egal, wie skeptisch man diesen gegenüber eingestellt sein mag, ,die Kategorie des Helden bleibt in einschlägigen umgangssprachlichen Topoi - den Gemeinplätzen plakativer heroisierender Selbstverständigung - , in Märchen, Mythen oder schließlich ausgreifenden politischen Ideationen virulent“" (Lipp 1985: 224f.).

Das Sprechen über die bislang medial erfolgte Charismatisierung, jetzt kritisch im Dienste einer De-Charismatisierung, geschieht vor allem über typische Identitätskategorien wie „Hoffnungsträger“, „Heilsbringer“, ,,neuer Typus von einem Politiker“, „Lichtgestalt“, ,starke Persönlichkeit“, „Bürgerkönig“, sowie Superlative („,beliebtester“), Intensivierer („,extrem hoch gelobt“), Präpositionalphrasen (,mit Sondereigenschaften“).

$\mathrm{Da}$ es schwierig ist, zu entscheiden, ob jemand, der wissenschaftlich unsauber arbeitet oder betrügt, auch ein schlechter Politiker ist, braucht es ein konkretes Ereignis, an dem sich direkt ablesen lässt, ob Guttenberg generell noch glaubwürdig ist. Pars pro toto referieren die Talkshow-Teilnehmer/innen auf ein mediales Ereignis am Vortrag und interpretieren es, stellen somit ein Diskursereignis her, das für sie Mustergültigkeit in punkto (mangelnder) Glaubwürdigkeit besitzt: Am 21.02.2011 tritt Guttenberg im Zuge eines Kommunalwahlkampfes auf und entschuldigt sich für die Fehler in seiner Dissertation. Aus dieser Rede, die bei YouTube unter dem Titel „Karl-Theodor zu Guttenberg (CSU) - 21.02.2011 „Stellungnahme zu Plagiatsvorwürfen" eingestellt wurde, wurde folgender relevanter Ausschnitt transkribiert:

[29], ,[...] Und das sind selbstverständlich Fehler. Und ich bin selbst auch ein Mensch mit Fehlern und Schwächen und deswegen stehe ich auch zu diesen Fehlern. Und zwar öffentlich zu diesen Fehlern, meine Damen und Herren. Und ich bin auch ganz gerne bereit, dies in die hier stehenden Kameras zu sagen, die de facto wegen der Kommunalwahl heute gekommen sind. Und ich sage ebenso, und das sage ich mit der notwendigen, und die mir in diesen Tagen gerne abgesprochenen Demut, auch die gehört zum politischen Handeln mit dazu, ich sage ebenso, dass ich mich von Herzen bei all jenen entschuldige, die ich mit Blick auf die Bearbeitung dieser Doktorarbeit verletzt habe. Das ist eine Entschuldigung, die von Herzen kommt, und die als solche auch zu sehen ist.“

(Guttenberg 2011)

Man könnte sagen, hier tritt ein Adliger auf, der sich selbst entzaubert, weil er öffentlich zugibt und betont, ein Mensch mit Fehlern zu sein - eine Selbststigmatisierung. Im fiktionalen Downton-Abbey-Kontext wäre dies geradezu ein Albtraum für jene Adligen, die ihr Charisma behalten wollen. Doch verglichen mit Uli Hoeneß, der zwar auch nur deskriptive Ausdrücke für seine 
Emotionen fand, der aber zugab, nachts schwitzend im Bett gelegen zu haben [11], gibt Guttenberg sehr reflektiert und metapragmatisch eine Lesart seiner Entschuldigung vor, nämlich, dass diese in Demut geschehe und „,von Herzen“ komme. Der Nachsatz ,und die als solche auch zu sehen ist", ist dabei nicht nur eine vorgegebene Lesart, sondern eine Aufforderung. Hier stellt sich nun die Frage, ob eine Selbstzuschreibung als „demütig“, die die Lesart der Interpretationsgemeinschaft explizit macht (,die mir in diesen Tagen gerne abgesprochenen Demut"), verbunden mit einem Vorschreiben der Perlokution der Handlung, auch zu einem geglückten Sprechakt führt.

\subsection{Authentizität I (personengebunden)}

Obwohl der Ausdruck „Authentizität“ im Gespräch über Guttenberg nicht verwendet wird, wird doch ausgehandelt, ob dieser sich bei seinem Auftritt authentisch verhalten habe. Dies wird im Folgenden an den hierfür relevanten Sequenzen rekonstruiert.

[21]

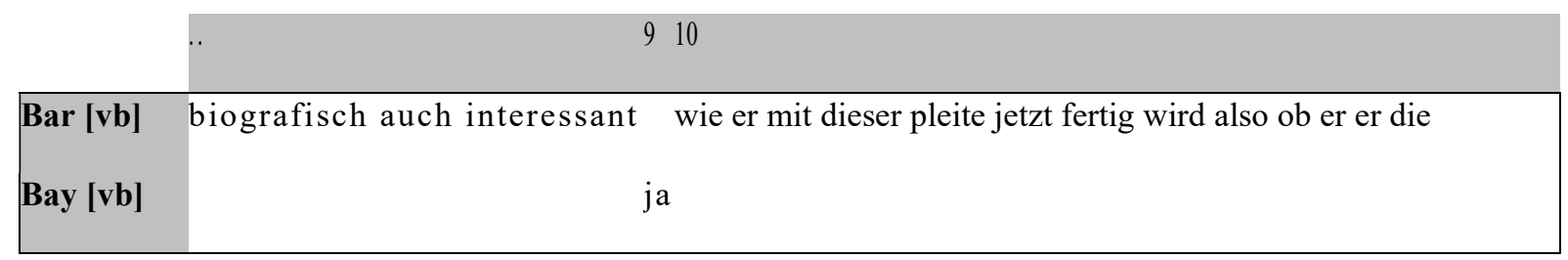

[22]
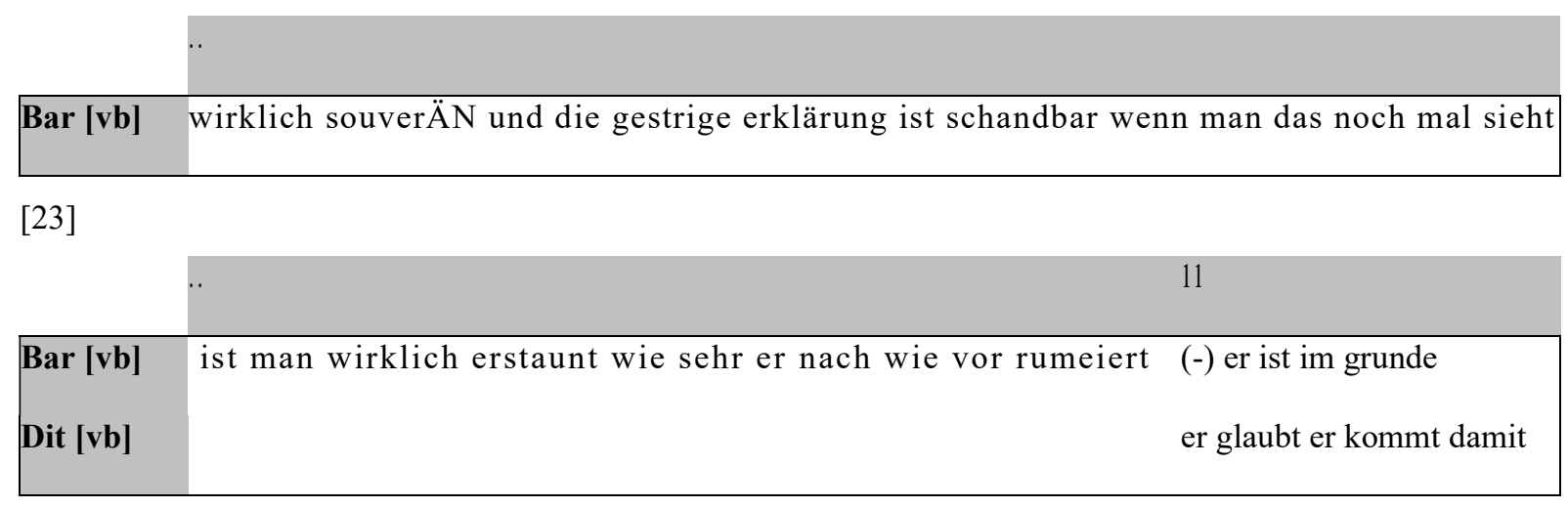

$[24]$

\begin{tabular}{llll|} 
& \multicolumn{1}{c}{12} & 13 & 14 \\
\hline Bar $[\mathbf{v b}]$ & genommen NAS Sforschwas er überHAUPT ist & er ist im grunde genommen von \\
Dit $[\mathbf{v b}]$ & durch & genau & \\
\hline
\end{tabular}

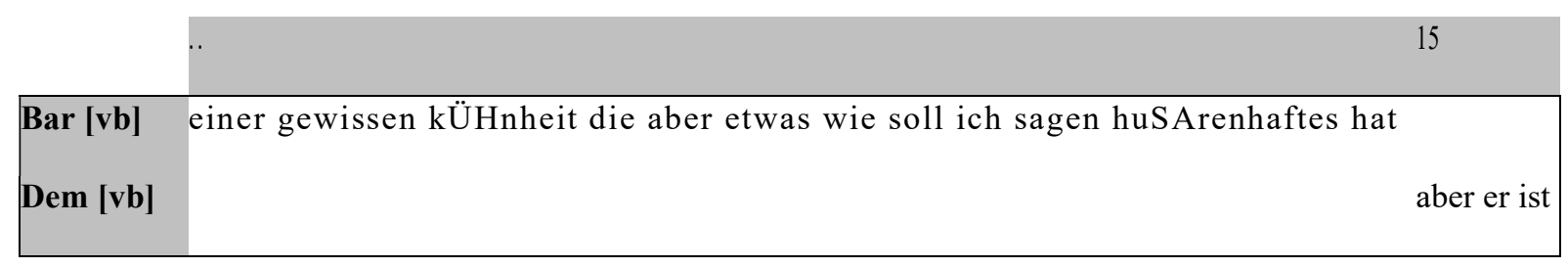

Für Baring, der hier als Entrüstungssubjekt auftritt, ist Guttenbergs Erklärung, der hier das abwesende Entrüstungsobjekt darstellt (cf. Christmann/Günthner 1999: 244), „schandbar“ (10). 
Das ist Guttenbergs aktuelle Verfehlung. Baring kritisiert Guttenbergs Verhalten als „rumeier[n]“ und als „NASSforsch“ (11). Interessant ist hier nun der Nachsatz „was er überhaupt ist" (12), mit dem verallgemeinernd Guttenbergs Art des Auftretens bezeichnet wird. Das Nassforsche, auch seine „KÜHnheit“, die mit „huSArenhaft[]“ heroisiert wird, referiert auf Guttenbergs gewohnte, typische Art des Auftretens (14). So gesehen trifft Baring vermutlich ins Schwarze, wenn er hier konstatiert, dass sich Guttenberg in seinem Auftreten treu geblieben ist und damit also in einer Weise agiert, die zuvor nicht nur von Guttenberg selbst als authentisch, sondern im Diskurs auch als charismatisch bezeichnet wurde. Auch die Journalistin Demmer konstruiert an anderer Stelle in der Maischberger-Talkrunde als weiteres Entrüstungssubjekt eine Kohärenz in der Art von Guttenbergs Auftreten:

all die probleme die er bislang hatte in seiner amtszeit als verteidigungsminister sind nach dem selben strickmuster abgelaufen (-) eigentlich hat er immer am anfang son bisschen geeiert (-) hat sich dann irgendwann positioniert und musste irgendwann die position wieder revidieren und hat immer gesagt man muss ja fehler machen dürfen (-) und kommt damit dann eben sehr wahrhaftig rüber (-) bislang waren halt die fehler die er gemacht hat sehr komplex (-) schwer zu kommunizieren (-) und jetzt ist es zum ersten mal sehr sehr sehr leicht zu verstehn und insofern auch zum ersten mal brenzlig.

(Demmer 2011)

Guttenbergs Auftreten sei „nach dem selben strickmuster abgelaufen“; das generalisierende Temporaladverb ,immer“ indiziert, dass das „eiern“ zu seinem bisherigen Stil gehört; wenn er dann Fehler zugegeben hat, kam er sehr ,wahrhaftig rüber“. Das Besondere an der neuen Situation sei nun, dass die Fehler nicht mehr zu vertuschen, sondern für jeden klar verständlich seien. Wenn Guttenberg also Fehler in seiner Doktorarbeit zugibt, und dies auf seine gewohnte, als authentisch wahrgenommene nassforsche Art, dann schadet ihm das nun. Mit anderen Worten: Nicht Guttenbergs Verhalten hat sich geändert, sondern die Umstände. Was hier implizit angesprochen wird, ist eine Art der medial bedingten Authentizität, insofern sich Guttenberg in den Medien in einem bestimmten Stil präsentiert hat. Diese Authentizität

- ist überzeitlich personengebunden

- ist interpretativ/sprachlich konstruiert

- indiziert die Wahrnehmung eines individuellen Verhaltens = individuelle medial inszenierte Authentizität $=\mathrm{z}$. B. nassforsch

- beschreibt eine kohärente diachrone Persönlichkeitsinszenierung á la „dem eigenen Stil treu bleiben“, unabhängig von sozialen und situativen Umständen

- führt zur Charismatisierung, wenn sie positiv evaluiert wird; im Fall von Guttenberg eine Ambivalenz aus sozialer Nähe und Distanz (,ein Adliger wie wir“)

Hier wird also aus der Perspektive einer konkreten Interpretationsgemeinschaft eine normative Form von Identität beschworen, die ähnlich in Bezug auf Charisma den Wesenskern von Menschen erfasst. Der Politiker muss „ein unverwechselbares Individualitätsprofil nicht nur darstellen, sondern von Sendung zu Sendung auch durchhalten können. “(Kugler/Kurt 2000: 153). Bei dieser Art personaler und vermuteter intrinsischer Authentizität wird erwartet, dass es eine „Übereinstimmung und Kohärenz zwischen dem gibt, was diese Person ,äußerlich`, also für andere wahrnehmbar, darstellt und ausdrückt und dem, was sie ,innerlich', also für sich selbst, ,tatsächlich“ ist"“ (Krämer 2012: 16). Guttenberg verhielt sich immer authentisch im Sinne einer 
authentischen Performanzfigur, nur wird diese authentische Art nun nicht mehr goutiert, weil sie nicht zu den situativen Umständen passt.

\subsection{Authentizität II (situationsgebunden)}

Guttenbergs Erklärung, die Art seines Auftretens, wird von den Akteuren weiter diskutiert und interpretiert. Dabei steht die Frage, ob er tatsächlich demütig aufgetreten ist, so, wie er es behauptet, oder ob sein Auftritt inauthentisch demütig war, im Zentrum der Aushandlung.

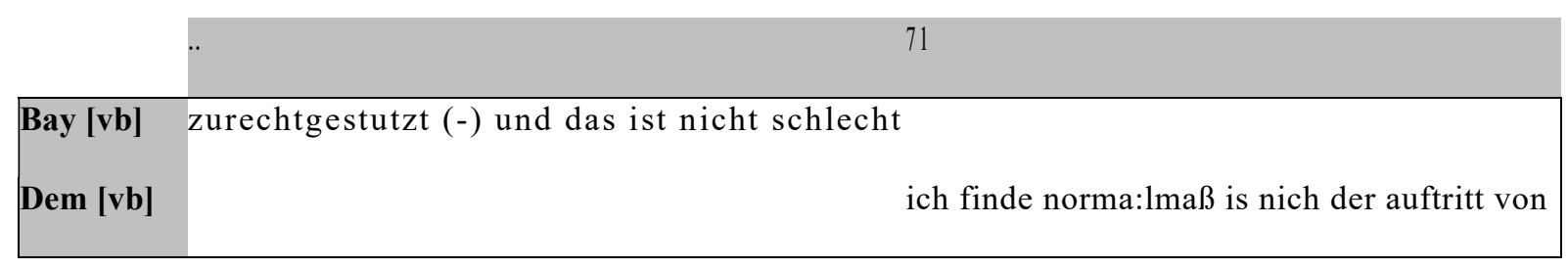

[111]
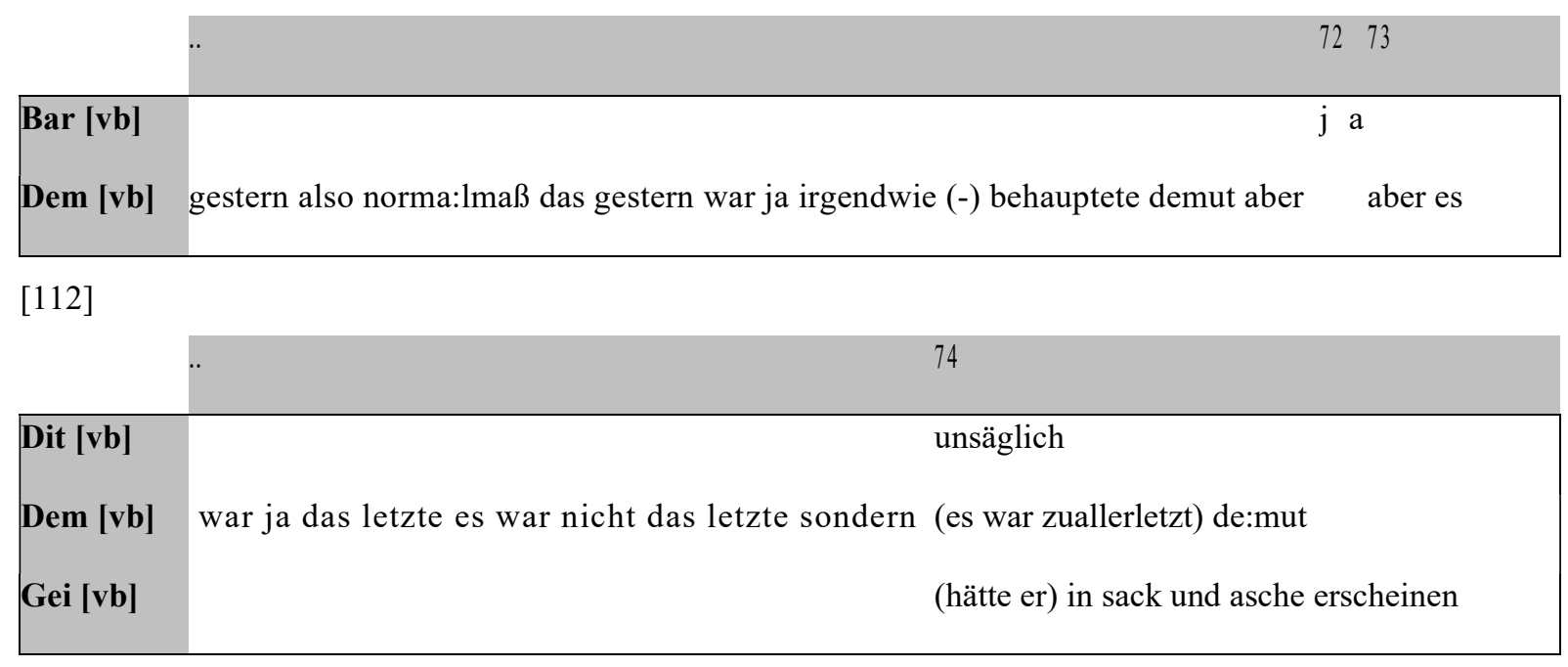

[113]

\begin{tabular}{|c|c|c|}
\hline & 75 & 76 \\
\hline \multicolumn{3}{|l|}{ Dit [vb] } \\
\hline Sch $[\mathbf{v b}]$ & & man könnte einen \\
\hline Dem [vb] & ne:in nicht in sack und asche aber de:mütig war es nunmal nich & \\
\hline Gei [vb] & sollen & \\
\hline
\end{tabular}

[114]

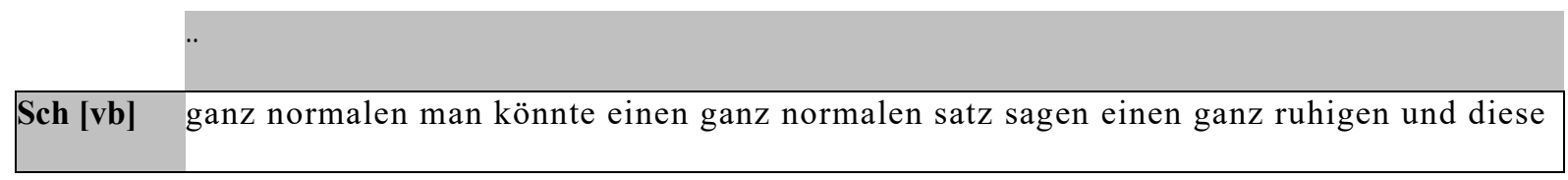




\begin{tabular}{|c|c|c|}
\hline & 7 & 78 \\
\hline Dit [vb] & & er ist ein opfer \\
\hline $\operatorname{Sch}[\mathbf{v b ]}$ & prätenTION mit der er da aufgetreten ist & \\
\hline Gei [vb] & \multicolumn{2}{|c|}{ er ist in einer schwierigen situation } \\
\hline
\end{tabular}

Es zeigt sich, dass die Aushandlung der Authentizität in mehreren Schritten verläuft, in denen verschiedene Diskursebenen angesprochen werden. Sie beginnt mit einer expliziten Positionierung Demmers mittels der Konstruktion ,,ich finde“ (71). Mit dieser wird vorab ein grundsätzlicher subjektiver Bewertungsmaßstab festgelegt. Weil Guttenberg bei dem Referenzereignis ihrem Anspruch auf „,norma:lmaß“ (71) nicht genügt, hier als eine Art der rhetorischen, aber auch moralischen Maßeinheit verstanden, wird der Aushandlungsprozess initiiert. Es muss an dieser Stelle festgehalten werden, dass die Aushandlung darüber, ob das Auftreten authentisch war oder nicht, eher eine Verhandlung, ein ,,mediales Tribunal“ in Abwesenheit des Delinquenten darstellt, der sich selbst nicht zu seiner eigenen Authentizität äußern darf - vor allem, weil er dies schon getan hat. Sich vorher oder hinterher über die eigene Authentizität zu äußern, ist nicht erlaubt; Authentizität bleib ein interpretatives und intersubjektives Konstrukt, es ist mit der betreffenden Person selbst nicht verhandelbar. Somit hat das eigene Verhalten, sobald es von jemandem, erst recht von einem selbst, auf den Prüfstand der Authentizität gestellt und mit situativen Umständen relational verknüpft wird, stets etwas Imagegefährdendes. ${ }^{8}$

Zunächst muss also ein Authentizitätszweifel konstruiert werden, der sich nur auf eine enttäuschte Erwartung beziehen kann. Es muss also auf der Merkmalsebene eine Vorstellung davon geben, was authentisches Verhalten gewesen wäre. Denn ob der Akteur im Inneren authentisch ist, kann äußerlich, kann epistemologisch nicht begründet werden. „Behaupten“ („,behauptete demut“ (1)) als ein Kommunikationsverb wird hier im Sinne von ,,nur behauptet, aber nicht gezeigt" gebraucht. Es wird also eine Diskrepanz zwischen einer deskriptiven und einer performativen Demut konstruiert.

Für Guttenberg Partei ergreifend kritisiert Geis die Angemessenheit in Demmers Ausdruckserwartung mittels metaphorischer Hyperbolik: ,hätte er in sack und asche erscheinen sollen“ (74). Diese Frage betrifft Demmers qualitative Ansprüche an ein authentisches Zeigen von Demut. Über die Redewendung spricht Geis die Zeitebene an, da er kontextualisiert, welche Erwartungen früher an Büßer gestellt wurden. Und diese Erwartungen an die rituelle Praxis, die erstmals im neuen Testament mit Buße in Verbindung gebracht wurde (cf. Röhrich 2001: 106), wäre heute, ebenso ein vergleichbares Auftreten, unzeitgemäß. Demmer beharrt auf ihrer Position und wiederholt diese auf der Zuschreibungsebene, eingeleitet durch eine adversative Konjunktion und mit der Konstruktion eines impliziten, verabsolutierenden Ausdrucksmaßstabes, abgeschwächt durch Abtönungspartikeln, ,aber de:mütig war es nun mal nicht“ (75). Sie gibt aber

\footnotetext{
${ }^{8}$ Cf. Lacoste/Leimgruber/Breyer (2014): „Authenticity may be argued to be a relational concept which accounts for the many ways in which a speaker or agent can be authentic in a given situation in relation to a particular aspect of his or her environment. Having this in mind, however, as Straub (2012: 10) has recently put it, authenticity ,comes with a warning that one should not buy into it without some good insurance'."
} 
keine Argumente für ihre These an. Ihre Position wird von Schneider geteilt und gestärkt, indem dieser nun auf der Merkmalsebene Authentizitätskriterien für das Zeigen von Demut angibt: Zur Demut gehört, einen ganz normalen und einen ruhigen Satz zu sagen (76), im Gegensatz dazu waren die Äußerungen von Guttenberg prätentiös. Geis gibt Guttenbergs schwierige Situation als erklärende Ursache dafür an, warum dieser sich in der Art äußert (77) und macht damit erneut seine Kontraposition deutlich. Es ist damit aber auch ein Eingeständnis, dass Guttenberg sich nicht so verhält, wie man es unter anderen, normalen Umständen von einem demütigen Politiker erwarten würde, wenngleich eine Haltung wie Demut wohl immer aus nicht normalen Umständen erwächst. Mit ihrem ironischen Gesprächsbeitrag „er ist ein opfer“ (78) lässt Ditfurth wiederum Geis’ Erklärung nicht gelten. Implizit zeigt sich darin der moralische Anspruch, dass derjenige, der die Situation verschuldet hat, sie auch wieder richten können muss bzw. sich zumindest dazu adäquat verhalten. Die Kategorie Opfer ruft zudem mit der Akteursebene das figurative Geflecht von sozialen Akteuren auf, die in den Fall eingebunden sind. Denn zu einem Opfer gehört immer auch ein Täter und vice versa. Guttenberg ist hier der Täter, die Gesellschaft, die getäuscht wurde, das Opfer.

Ausgehandelt wurde in diesem kurzen Sprecherwechsel nun eine andere Art von Authentizität, die zwar personengebunden ist, weil sie sich auf den Akteur Guttenberg und sein Verhalten bezieht, die aber darüber hinaus situationsgebunden ist, wie dies für die situative Differenzfigur typisch ist. Es geht um ein konkretes Verhalten in einer konkreten Situation und nicht um einen generellen Stil, mit dem jemand kohärent erscheint. Diese Art von Authentizität

- ist situations- und personengebunden

- ist interpretativ/sprachlich konstruiert, kommunikativ ausgehandelt

- indiziert die Wahrnehmung eines individuellen Verhaltens (Authentizität I)

- beschreibt eine ritualisierte, medial inszenierte Authentizität (z. B. Demut, Dankbarkeit, Höflichkeit) und damit einen angemessenen Ausdruck erwarteter Gefühle/Haltungen

- beinhaltet sozial anerkannte Authentizitätsmarker, abhängig von sozialen und situativen Umständen = sozial adäquate, synchrone Persönlichkeitsinszenierung

- führt bei einer negativen Evaluation zur De-Charismatisierung (Vertrauensverlust oder Entindividualisierung)

Angemessen wäre für die Akteure in diesem Fall ein Verhalten gewesen, das man als „echte Demut", nicht aber als „echten Guttenberg“ hätte verifizieren können. Normativ ist hier einerseits die Erwartung der richtigen Haltung (nämlich Demut, die Guttenberg antizipierend verbalisiert) und andererseits die Erwartung des dazu richtigen Ausdrucks. Es braucht Authentizitätsmarker, die ebenfalls ausgehandelt werden, an denen man erkennen kann, ob jemand das Ritual des Demutzeigens auf korrekte Weise realisiert. Bei Ritualen, auch bei politischen Demutsritualen, kommt es nicht darauf an, welche innere Haltung die Teilnehmer/innen tatsächlich haben: „Im Ritual werden Handlungen nicht ausgewählt, um Intentionen zu verwirklichen oder auszudrücken. Solange man die Regeln befolgt, gibt es keinen Anlass, die Handlung deswegen zu korrigieren, weil sie nicht das ist, was man meinte." (Humphrey/Laidlaw 2013: 143). Nun ist es im Fall von Guttenberg strittig, ob er die Regeln für eine verbale ritualisierte Demutsgeste befolgte, die den Ansprüchen an Authentizität genügt. Dass Guttenberg seine innere Haltung verbalisiert und das Ritual explizit macht (,,auch die gehört zum politischen Handeln mit dazu“"), hat die intersubjektiven Ansprüche erst evoziert. Hätte er sich nur entschuldigt, 
hätten andere zumindest nicht behaupten können, er wäre dem Demutsritual nicht gerecht geworden, da er selbst dieses Ritual nicht als Interpretationsrahmung (cf. Goffman 1980) verfügbar gemacht hätte. Deshalb ist die entscheidende Frage, ob sich das Unangemessene in Guttenbergs Auftreten für die Akteure nicht gerade darin zeigt, dass es zu sehr nach Ritual und zu wenig nach persönlichem Ausdruck aussah. Aber eben das fordert „die Intimgesellschaft“, schreibt Han. Sie

misstraut den ritualisierten Gesten und dem zeremoniell geformten Verhalten. Ihr erscheinen sie als äußerlich und inauthentisch. Das Ritual ist eine Handlung aus externalisierten Ausdrucksformen, die ent-individualisierend, ent-personalisierend und ent-psychologisierend wirken. Die daran Beteiligten ,sind expressiv', ohne jedoch sich selbst zur Schau stellen oder entblößen zu müssen. Die Intimgesellschaft ist eine psychologisierte, entritualisierte Gesellschaft. Sie ist eine Gesellschaft des Geständnisses, der Entblößung und der pornografischen Distanzlosigkeit.

(Han 2013: 60, Hervorhebungen i. O.).

Guttenbergs Entschuldigung war vermutlich zu gefasst und zu wenig ein „Schwitzen“ und „Wälzen“ in der eigenen Schuld à la Hoeneß. Oder man kann auch sagen: „Die kollektiven Gefühle, aufs Höchste aufgebracht, verlangen Genugtuung“ (Hondrich 2002: 16), sind aber durch Guttenbergs Performance nicht befriedigt worden: „Verletzte Werte müssen geheilt, unscharfe Regeln verschärft, hochgestiegene Karrieristen gestürzt, Individuen geopfert werden auf dem Altar der von vielen geteilten moralischen Gefühle." (Hondrich 2002: 16). Dabei ist das Zeigen von Demut als politisches Ritual selbst bereits eine Perversion der ursprünglichen Idee von Demut, die nichts Äußerliches ist, sondern ein rein innerliches Phänomen, ,als innere Form des Habitus selbst“ (Hartmann 1962: 476). „Demut empfindet der Mensch nicht vor den Menschen“ schreibt Hartmann in seiner „Ethik“, „,das wäre falsche Demut, Selbsterniedrigung, sklavischer Sinn“. „Alle demütige Haltung nach außen ist im Grunde ein falsches Ethos - es birgt sich immer irgendein moralischer Defekt in ihr, ein Irrewerden an sich, Kleinmut, Verängstigung, oder gar ein unechtes Pathos, falsche Scham, Menschenfurcht.“ (Hartmann 1962: 476f.) Insofern ist bereits die Erwartung, jemand solle echte Demut zeigen, eine unmögliche Bedingung für authentische Demut. Und wenn jemand von sich selbst meint behaupten zu müssen, er zeige Demut, in explizitem Kontrast zu der Erwartung der anderen, er zeige keine Demut, dann ist dies eine reflexive Form inauthentischer Demut.

In Bezug auf sein Charisma lässt sich sagen, dass Guttenberg es bezogen auf diese Situation wohl nur verlieren konnte. Führte früher seine individuelle nassforsche Art zur Charismatisierung, so konfligiert sie nun mit der rahmenden Demut. Denn Demut ist aus Sicht der Interpretationsgemeinschaft 1. nicht „,nassforsch“, sondern vielleicht bescheiden, zurückhaltend, gradlinig und nicht „eiernd“, und 2. wird niemand als etwas Besonderes charismatisiert, wenn er angepasst Rituale bedient. Entweder ist das Charisma in Gefahr aufgrund einer rituellen Entindividualisierung (zumal beim Eingeständnis einer Schuld) oder, wenn das ,authentische rituelle"Verhalten zu stark ritualisiert ist, aufgrund von Vertrauensverlusten.

Ditfurth gibt in der Talkshow-Runde eine allgemeine soziale Erklärung dafür, warum Guttenberg generell nicht authentisch und schon gar nicht demütig auftreten könne. Dies habe mit seiner adligen Erziehung zu tun: 
zur erziehung gehört auch die überlegenheit nie zu zeigen die wird nur intern besprochen (-) das ist genau das spiel

(Ditfurth 2011)

jeden tag saugt er das auf und die arroganz in seiner sogenannten demütigen entschuldigung trifft ja auch nie den punkt deswegen

(Ditfurth 2011)

Authentisch ist mithin bei Guttenberg ohnehin nur das inauthentische Adlige. Authentizität I wird also zur Ursache für mangelnde Authentizität II. An Ditfurths pauschalisierendem Urteil wird einmal mehr deutlich, dass Authentizität „,keine deskriptive Eigenschaft [ist], deren Vorliegen bloß festzustellen wäre. Sie ist immer eine moralische Qualität.“ (Deppermann 2000: 239).

\subsection{Authentizität III (ideal)}

Guttenbergs soziale Identität wird verhandelt, wenn darüber diskutiert wird, wie er zukünftig in seiner sozialen Rolle als Politiker auftreten sollte. Zugrunde gelegt wird dabei jedoch Guttenbergs bisherige personale Identität. Wir sehen hier, was Coupland (2014: 19) konstatiert: „Authenticity also surfaces at the interface between personal and social identities, in our relationships with others and in our allegiances to groups and communities, at whatever level of generality. “

Baring zieht daraus, dass der ,nassforsche“ Stil Guttenbergs nun nicht mehr ankommt, die Konsequenz, dass dieser den „Stil, den er hat“ überprüfen müsse.

wenn er sich nicht neu erfindet zu dem bescheideneren demütigeren nicht mehr so hoch fahrenden wenn er sozusagen ein guttenberg 2 wird (-) würd ich sagen dann würd er überleben

(Baring 2011)

Bemerkenswert ist hier die Formulierung ,wenn er sich nicht neu erfindet“, die indiziert, dass es nicht nur darum geht, äußerlich anders aufzutreten, sich anders zu inszenieren, sondern auch um eine Transformation im Inneren. Letztlich ist eine solche Identitätstransformation nicht überprüfbar, weil eine Person lediglich ihr Auftreten und damit nur ihr Image im Sinne eines Eindrucksmanagements ändern kann (cf. Goffman 1999). Es käme nur darauf an, dass dieses Image so inszeniert wird, dass es als ein authentisches Selbst erscheint. In beiden Fällen, wenn Guttenberg seine Identität nur äußerlich oder aber tatsächlich, angepasst an die Erwartungen, ändern würde (als wäre dies so einfach), hätte diese neue Identität, der ,guttenberg 2“, der ein bisschen nach „Terminator 2“ - der neuen Bauweise eines Roboters - klingt, mehr von einem Trickster, dessen Metier der Gestalt- und Identitätswandel zum eigenen Vorteil ist (,dann würd er überleben") (cf. Steen 2015b), als von einer authentischen persönlichen Entwicklung. Rosa (2016) kritisiert derartige spätmoderne Ansprüche an die Entwicklung des Individuums folgendermaßen:

Unter den Bedingungen der hochdynamischen Spätmoderne kollidiert der aus dem Authentizitätsgedanken resultierende Auftrag, herauszufinden, wer wir wirklich sind, mit der sozialstrukturell erzeugten Zumutung, uns immer wieder „neu zu erfinden“ und kreativ neu zu bestimmen. Dass auch diese Neuerfindung noch „ganz authentisch“ erfolgen soll, gehört zu den zugespitzten Paradoxien der Gegenwart. 
Auf die Zukunft gerichtet, wird von Guttenberg also eine „neue“ Authentizität im Verhalten erwartet. Dabei tritt Baring als Autorität auf, die festlegen kann, wie diese sich performativ äußern soll. Diese verlangte Art der Authentizität

- ist situationsgebunden und personengebunden, normativ/ideal

- ist sprachlich konstruiert, vorab sozial/kommunikativ ausgehandelt

- indiziert eine Erwartung der Wahrnehmung eines echten individuellen Verhaltens („Authentizität I“), das gleichzeitig den Wünschen der Interpretationsgemeinschaft nach ritueller Angemessenheit entspricht (,Authentizität II“)

- beschreibt sozial anerkannte Authentizitätsmarker, die individualisiert verwendet werden, abhängig von sozialen und situativen Umständen = sozial adäquate, diachrone Persönlichkeitsinszenierung, die individuell und authentisch erscheint (bescheidener, demütiger, nicht mehr so hochfahrend)

- führt bei einer positiven Evaluation zur Re-Charismatisierung (?)

Beim idealen authentischen Verhalten werden situative Performanzkriterien und der unverwechselbare personale Stil so vereint, dass das individuelle Verhalten automatisch ein intersubjektiv angemessenes ist. Dann aber würde wohl der Politiker, wie Guttenberg es ausdrückte, sich den „Fliehkräften derer [...] unterwerfen, die versuchen, einen irgendwo einzunorden“ [26]. Oder wie Deppermann (2000: 244) es ausdrückt: „Man ist eben gerade dann nicht authentisch, wenn man nur deshalb authentisch ist, weil man es sein soll." ${ }^{\text {"9 }}$

Nach der Verhandlung in der Talkshow greift die Presse immer wieder Guttenbergs inauthentische Demut auf:

[30] „Dick aufgetragene Demut“

(Löwenstein 2011, Frankfurter Allgemeine)

[31] „Guttenbergs falsche Demut“

(Polke-Majewski 2011, Die Zeit)

[32] „Aus dem Ausland greift der einst populärste CSU-Politiker Guttenberg seine eigene Partei an und verlangt in Sachen Plagiat Verzeihung. Er übt Reue, aber ohne große Demut.“

(Krauel 2011, Welt)

[33] „In seiner Mail schrieb Guttenberg von ,berechtigten Gründen“ für seinen Rücktritt und ,meinen lausigen Umgang، damit. Dass viele dahinter jetzt die Inszenierung eines Bußschauspiels vermuten, hat er sich mit der Überinszenierung seiner Erstkarriere selbst eingebrockt. Zugleich sollte man seine Worte schon als das nehmen, was sie sind: ein erster Versuch umfassender Demut. Selbstkritik bei Guttenberg hieß bisher, sich neben sich zu stellen und über den eigenen ,Blödsinn“ zu staunen. Jetzt hat er eine neue Stufe der Reue erreicht: ein

\footnotetext{
${ }^{9}$ Es geht hier also um einen utilitaristischen Nutzen von Authentizität und nicht etwa um einen persönlichen, wie ihn etwa der amerikanische Psychologe Rogers entwirft: „Wenn die Menschen lernten, nur auf sich selbst, auf ihre inneren Werte zu hören, entdeckten sie die, Quellen für das gute Leben in sich selbst, nicht in einem äußeren Dogma oder Diktum oder in irgend etwas Materiellem“."(Dietschi 2012: 27; Rogers 2007: 170). In den Worten Rosas (2016) wird hier ein stummes, kein resonantes Verhältnis zur sozialen Welt gefordert. Denn es wird „ein Resonanzverhältnis [...] dadurch verunmöglicht, dass sich das Subjekt zu radikal öffnet oder an die Welt verliert: Es büßt dann gleichsam seine ,Eigenfrequenz' ein, es spricht nicht mehr mit eigener Stimme, sondern wird konturlos, indem es nur noch als ,Echo“ der Welt fungiert.“(Rosa 2016: 191).
} 
Schuldeingeständnis, dem nicht gleich ein trotziges ,aber" folgt.“

(Deininger 2016, Süddeutsche Zeitung)

Als Guttenberg am 23.09.2016 in Berlin auftrat und dort noch einmal seinen Rücktritt und seinen Umgang mit den Medien reflektierte, interpretierte und bewertete man dies folgendermaßen:

[34] „Mit Hipster-Brille, Bart und azurblauem Sakko, rhetorisch geschliffen und selbstkritisch bis zur Koketterie tritt der einstige Hoffnungsträger der CSU in Berlin vor die Teilnehmer einer Konferenz."

$\left(\mathrm{NN}_{4} 2016\right.$, Welt $)$

Nach Re-Charismatisierung klingt dies alles nicht und auch nicht nach der Beurteilung eines authentischen ,selbstkritischen“ Verhaltens. Denn wer kokettiert [34], der rückt im Zuge einer sowieso ,allgegenwärtigen Tendenz zur Selbstoffenbarung“ nicht „was, sondern dass gefühlt wird“ (Krämer 2012: 17, Hervorhebungen i. O.) oder hier, dass etwas reflektiert wird, ins Zentrum seines Auftritts - ,authentisch geht anders“. Vielmehr scheint sich bis dato im Diskurs über den Begriff der Demut verfestigt zu haben, dass Guttenbergs Stil (Authentizität I) generell ein inauthentischer ist.

Es wird deutlich, dass Authentizität eine wichtige rhetorische Ausschluss- und Bewertungsoperation darstellt (cf. Deppermann 2000: 248). Obwohl der Ausdruck nicht explizit genannt wird, ist Authentizität - also die Beurteilung, ob jemand z. B. tatsächlich „,demütig“ oder nur „,behauptend demütig“ auftritt, ob er tatsächlich „,selbstkritisch“ oder zu reflektiert und anbiedernd selbstkritisch auftritt, ein wichtiges Kriterium dafür, ob dieser Politiker noch oder wieder ein glaubhafter Politiker ist, der sein Amt behalten oder in der deutschen Politiklandschaft Fuß fassen darf: „Authenticty matters. It remains a quality of experience, that we actively seek out, in most domains of life, material and social. [...] We value authenticity and we tend to be critical of pseudo-authenticity.“ (Coupland 2003: 417).

\section{$4 \quad$ Zusammenfassung}

Sowohl die exemplarischen Diskursanalysen als auch das Fallbeispiel Guttenberg, bei dem Diskurs und Gespräch aufeinander bezogen wurden, zeigen, dass Authentizität ,ein Medium der Sozialität“ ist, „das für das gesellschaftliche Leben von Belang ist" (Krämer 2012: 25). Die Konzepte Authentizität und Charisma werden im Diskurs vielfach auf ähnliche Weise konstruiert, vor allem die Diskursebenen Akteure, Zuschreibung, System und Zeit betreffend. Hier zeigte sich die von Knaller/Müller (2006: 11) postulierte Nachbarschaft der Begriffe. Unterschiede konnten festgestellt werden hinsichtlich der Diskursereignisebene in Verbindung mit konstruierten Krisen sowie der Merkmalsebene, da sich Charisma häufig als positive Performanzfigur beschreiben lässt, Authentizität als negative Differenzfigur. Beide ergänzen sich jedoch: Der Politiker wird charismatisch dadurch, dass er etwas anders macht als es der Politikbetrieb erwartet und dadurch weniger den Routinen und Imagespielen unterworfen wirkt, sondern eben autonomer, authentischer. Das Beispiel Guttenberg hat wiederum gezeigt, dass diese Rechnung nicht aufgeht, sobald es um das Eingestehen von Niederlagen oder Schuld geht. Denn wenn der Eindruck einer personalen (sogar ehemals als charismatisch empfundenen) Authentizität mit einer geforderten rituellen Authentizität kollidiert, befindet sich die Person in einem 
Zwiespalt aus Individualität und Ritualität. Das charismatische Abweichen von der Routine oder aber das explizite Hinweisen auf diese führt dann nicht zur Wahrnehmung von Authentizität. Im Gegenteil, es überwiegt der Eindruck, der Person sei die rituelle Ordnung egal, und die Irritation der Ordnung führt vorerst nicht zur Charismatisierung, sondern zur Stigmatisierung, oder aber es gehe nur um die rituelle Ordnung und nicht um Individualität. Eine nur scheinbare Lösung ist die aus der Dialektik von Individualität und Ritualität hervorgehende angemessene Authentizität als ein noch zu verwirklichendes Ideal. Somit hat das Beispiel Guttenberg gezeigt, dass es nicht nur eine Sehnsucht nach Authentizität gibt, sondern dass diese Authentizität auch angemessen sein soll. Das geht nicht immer ohne Widersprüche: „Die Attribution und Assoziation von Bedeutungen und Erwartungen an die Rolle, die von den politischen Repräsentanten ausgefüllt werden soll, sind vielfältig und keinesfalls widerspruchsfrei.“ (Jentges 2010: 78). Angemessenheit hat dann immer auch etwas mit Macht zu tun, insofern es von Belang ist, wer bestimmt, was zu welcher Zeit angemessen ist. Die Aushandlung von $\mathrm{Au}$ thentizität im Gespräch zeigt, dass „Vorstellungen von Echtheit, Eigentlichkeit, Unmittelbarkeit gesellschaftlich bedingte, kontingente Konstrukte sind, die kommunikativ generiert und im Rahmen von Machtbeziehungen verhandelt werden.“ (Rössner/Uhl 2012: 9).

\section{Literaturverzeichnis}

Baudrillard, Jean (1978): „Präzession der Simulakra“. In: Baudrillard, Jean (ed.): Agonie des Realen. Berlin, Merve: 7-71.

Baumgart, Ralf/Eichener, Volker (1997): Norbert Elias zur Einführung. 2. Aufl. Hamburg: Junius.

Christmann, Gabriele B./Günthner, Susanne (1999): „Entrüstung: Moral mit Affekt““. In: Bergmann, Jörg/Luckmann, Thomas (eds.): Kommunikative Konstruktion von Moral. Bd. 1: Struktur und Dynamik der Formen moralischer Kommunikation. Opladen, Westdeutscher Verlag: 242-274.

Coupland, Nikolas (2003): "Sociolinguistic authenticities". In: Journal of Sociolinguistics 7/3: 417-442.

Coupland, Niklas (2014): „Language, society and authenticity. Themes and perspectives“. In: Lacoste, Véronique/Leimgruber, Jakob/Breyer, Thiemo (eds.): Indexing Authenticity. Sociolinguistic Perspectives. Berlin/Boston, de Gruyter: 14-39.

Deppermann, Arnulf (2000): Authentizitätsrhetorik: „Sprachliche Verfahren und Funktionen der Unterscheidung von ,echten“ und ,unechten“ Mitgliedern sozialer Kategorien“. In: Eßbach, Wolfgang (ed.): Identität und Alterität in Theorie und Methode. Würzburg, Ergon: 231-252.

Dietschi, Daniel (2012): Hinführungen zur Authentizität. Die ideen- und begriffsgeschichtliche Aufarbeitung eines modernen Persönlichkeitsideals. Würzburg: Königshausen \& Neumann.

Elias, Norbert (1997): Über den Prozeß der Zivilisation. Soziogenetische und psychogenetische Untersuchungen. Bd 1. Wandlungen des Verhaltens in den weltlichen Oberschichten des Abendlandes. Frankfurt a. M.: Suhrkamp.

Fauconnier, Gilles (1997): Mappings in Thoughts and Language. Cambridge: Cambridge University Press. 
Felder, Ekkehard (2013): „Der diskursive Wettkampf um Geltungsansprüche“. In: Felder, Ekkehard (ed.): Faktizitätsherstellung in Diskursen. Die Macht des Deklarativen. Berlin/Boston, de Gruyter: 13-28. (= Sprache und Wissen, Bd. 13).

Ferrara, Alessandro (1998): Reflective Authenticity. Rethinking the project of modernity. London/New York: Routledge.

Goffman, Erving (1980): Rahmen-Analyse. Ein Versuch über die Organisation von Alltagserfahrungen. Frankfurt a. M.: Suhrkamp.

Goffman, Erving (1999): Interaktionsrituale. Über Verhalten in direkter Kommunikation. 5. Aufl. Frankfurt a. M.: Suhrkamp.

Han, Byung-Chul (2013): Transparenzgesellschaft. 3. Aufl. Berlin: Matthes \& Seitz.

Harré, Rom/van Langenhove, Luk (1999): Positioning Theory. Moral Contexts of Intentional Action. Oxford: Blackwell.

Hartmann, Nicolai (1962): Ethik. 4. Aufl. Berlin: de Gruyter.

Hermanns, Fritz (1995): „Kognition, Emotion, Intention. Dimensionen lexikalischer Semantik“. In: Harras, Gisela (ed.): Die Ordnung der Wörter. Berlin/New York, de Gruyter: $138-178$.

Hondrich, Karl Otto (2002): Enthüllung und Entrüstung. Eine Phänomenologie des politischen Skandals. Frankfurt a. M.: Suhrkamp.

Humphrey, Caroline/Laidlaw, James (2013): „Die rituelle Einstellung“. In: Belliger, Andréa/Krieger, David J. (eds.): Ritualtheorien. Ein einführendes Handbuch. 5. Aufl. Wiesbaden, Springer: 133-153.

Jentges, Erik (2010): Die soziale Magie politischer Repräsentation. Charisma und Anerkennung in der Zivilgesellschaft. Bielefeld: transcript.

Keller, Rudi (1994): Sprachwandel. 2. Aufl. Tübingen/Basel: Francke.

Kienpointner, Manfred (1992): Alltagslogik. Struktur und Funktion von Argumentationsmustern. Stuttgart/Bad Cannstatt: Frommann.

Knaller, Susanne/Müller, Harro (2005): „Authentisch/Authentizität“. In: Barck, Karl-Heinz et al. (eds.): Ästhetische Grundbegriffe. Historisches Wörterbuch in sieben Bänden. Bd. 7. Stuttgart, J. B. Metzler: 40-65.

Knaller, Susanne/Müller, Harro (2006): „Einleitung“. In: Knaller, Susanne/Müller, Harro (eds.): Authentizität. Diskussion eines ästhetischen Begriffs. München, Fink: 7-11.

Krämer, Sybille (2012): „Zum Paradoxon von Zeugenschaft im Spannungsfeld von Personalität und Depersonalisierung. Ein Kommentar über Authentizität in fünf Thesen“. In: Rössner, Michael/Uhl, Heidemarie (eds.): Renaissance der Authentizität? Über die neue Sehnsucht nach dem Ursprünglichen. Bielefeld, trascript: 15-27.

Kugler, Christine/Kurt, Ronald (2000): „Inszenierungsformen von Glaubwürdigkeit im Medium Fernsehen. Politiker zwischen Ästhetisierung und Alltagspragmatik“. In: FischerLichte, Erika/Pflug, Isabel (eds.): Inszenierung von Authentizität. Theatralität. Tübingen/Basel, Francke: 149-162.

Lacoste, Véronique/Leimgruber, Jakob/Breyer, Thiemo (2014): „Authenticity: A view from inside and outside sociolinguistics". In: Lacoste, Véronique/Leimgruber, Jakob/Breyer, Thiemo (eds.): Indexing Authenticity. Sociolinguistic Perspectives. Berlin/Boston, de Gruyter: $1-13$. 
Lenze, Malte (2002): Postmodernes Charisma. Marken und Stars statt Religion und Vernunft. Wiesbaden: Deutscher Universitäts-Verlag.

Link, Jürgen (1983): „Kollektivsymbolik und Mediendiskurse“. kultuRRevolution 1: 6-21.

Lipp, Wolfgang (1985): Stigma und Charisma. Über soziales Grenzverhalten. Berlin: Reimer.

Noetzel, Thomas (1999): Authentizität als politisches Problem. Ein Beitrag zur Theoriegeschichte der Legitimation politischer Ordnung. Berlin: Akademiverlag (= Politische Ideen, Bd. 9).

Röhrich, Lutz (2001): Lexikon der sprichwörtlichen Redensarten. Bd. 1, 5. Aufl. Freiburg: Herder.

Rössner, Michael/Uhl, Heidemarie (2012): „Vorwort“. In Rössner, Michael/Uhl, Heidemarie (eds.): Renaissance der Authentizität? Über die neue Sehnsucht nach dem Ursprünglichen. Bielefeld, transcript: 9-14.

Rogers, Carl (2007): Der neue Mensch. 8. Aufl. Stuttgart: Klett-Cotta.

Rosa, Hartmut (2016): Resonanz. Eine Soziologie der Weltbeziehung. Berlin: Suhrkamp.

Selting, Margret et al. (2009): „Gesprächsanalytisches Transkriptionssystem 2. (GAT 2)“. Gesprächsforschung - Online-Zeitschrift zur verbalen Interaktion 10/2009: 353-402. (= www.gespraechsforschung-ozs.de)

Sennett, Richard (2004): Verfall und Ende des öffentlichen Lebens. Die Tyrannei der Intimität. 14. Aufl. Frankfurt a. M.: Fischer.

Spitzmüller, Jürgen/Warnke, Ingo (2011): Diskurslinguistik. Eine Einführung in Theorien und Methoden der transtextuellen Sprachanalyse. Berlin/Boston: de Gruyter.

Steen, Pamela (2015a): „,Charisma macht müde Demokratien munter““ - Zum diskursiven Zusammenhang von Charisma und Demokratie“. In: Knobloch, Clemens/Vogel, Friedemann (eds.): Sprache und Demokratie. 73, 4. Linguistik Online: https://doi.org/10.13092/1o.73.2198.

Steen, Pamela (2015b): Die kommunikative Identität des Tricksters. Eine gesprächslinguistische und kultursemiotische Untersuchung zur Identitätskonstruktion in einer marginalisierten Gruppe. Heidelberg: Winter. (= Oralingua 10).

Steen, Pamela (2017): „Charisma“. In: Lasch, Alexander/Liebert, Wolf-Andreas (eds.): Handbuch Sprache in der Religion. Berlin/Boston, de Gruyter: 288-311. (= HSW 18).

Straub, Julia (2012): „Introduction: The paradoxes of authenticity“. In: Straub, Julia (ed.): Paradoxes of Authenticity. Studies on a Critical Concept. Bielefeld: transcript.

Thornborrow, Joanna/Leeuwen, Theo van (eds.) (2001): Authenticity in Media Discourse. Thematic Issue of Discourse Studies 3/4.

Ulrich, Anne (2012): „Authentizität“. In: Ueding, Gerd (ed.): Historisches Wörterbuch der Rhetorik. Bd. 10., Nachträge A-Z., Berlin/Boston, de Gruyter: 79-91.

Weber, Max (1976): Wirtschaft und Gesellschaft. Grundriss der verstehenden Soziologie. 5. Aufl. Tübingen: J. C. B. Mohr.

Weibler, Jürgen (2010): „Obama kam, sprach und siegte - Oder wie Reden Führung begründen“. In: Weibler, Jürgen (ed.): Barack Obama und die Macht der Worte. Wiesbaden, VS Verlag: $12-38$. 


\section{Verzeichnis der Internet-Quellen}

Burmester, Hanno (2014): „Charisma macht müde Demokratien munter“. 06.10.2014 Berliner Republik.de. b-republik.de/archiv/charisma-macht-muede-demokratien-munter [04.11.2020].

Deininger, Roman (2016): „Guttenberg übt sich in Demut - oder ist es Kalkül?“ Süddeutsche Zeitung.de 01.03.2016 sueddeutsche.de/bayern/csu-guttenberg-uebt-sich-in-demut1.2885217 [04.11.2020].

Denkler, Thorsten/Jakat, Lena (2011): „Fleißiger Alleinunterhalter, sozialer Anti-Guido.“ Süddeutsche Zeitung.de 05.04.2011. sueddeutsche.de/politik/fdp-sechs-thesen-ueber-philipproesler-fleissiger-alleinunterhalter-sozialer-anti-westerwelle-1.1081250 [04.11.2020].

Denkler, Thorsten (2015): „Selfie-Time für Twitter-Könige“. Süddeutsche Zeitung.de 01.04.2015. sueddeutsche.de/politik/politiker-in-den-sozialen-medien-selfie-time-fuer-twitter-koenige-1.2406263 [04.11.2020].

Eichhorn, Moritz (2013): „Was steht denn da auf dem Zettel seiner Frau?“ Bild.de 14.06.2013 bild.de/politik/inland/maybrit-illner/ehefrau-bereitete-steinbrueck-auf-talkshow-vor30840542.bild.html [04.11.2020].

Esslinger, Detlef (2013): „Deutschland, einig Prangerland“. Süddeutsche Zeitung.de 0505.2013. sueddeutsche.de/politik/skandale-in-der-mediengesellschaft-deutschland-ein-pran gerland-1.1665130 [04.11.2020].

Fücks, Ralf (2012): „Kleinstes Karo statt großes Kino“. Süddeutsche Zeitung.de 05.01.2012. sueddeutsche.de/politik/zum-interview-von-christian-wulff-kleinstes-karo-statt-grosseskino-1.1251470-0 [04.11.2020].

Hebestreit, Steffen (2013): „Irrtum im Amt““. 10.05.2013. fr.de/politik/irrtum-11279294.html [04.11.2020].

Hildebrandt, Tina (2010): „Abgeblitzt und abgeschossen“. Zeit.de 01.07.2010. zeit.de/2010/27/Politiker [04.11.2020].

Hofmann, Gunter (2009): „Hört! Hört“ Zeit.de 03.09.2009. zeit.de/2009/37/60-Jahre-Bundes$\operatorname{tag}$ [04.11.2020].

Jakobs, Hans-Jürgen (2010): Schlossgespenst der Politik. Süddeutsche Zeitung.de 28.10.2010. sueddeutsche.de/medien/phaenomen-guttenberg-das-schlossgespenst-der-deutschen-politik-1.1016928 [04.11.2020].

Körner, Torsten (2013): „Das Verzwergen der Helden“. Tagesspiegel.de 17.08.2013. tagesspiegel.de/themen/digitalisierung-ki/charisma-in-zeiten-des-internet-das-verzwergen-der-helden/8651514.html [04.11.2020].

Kornelius, Stefan (2010a): „Wahlkampf in Washington“. Süddeutsche Zeitung.de 17.05.2010. sueddeutsche.de/politik/merkel-besucht-obama-wahlkampf-in-washington-1.111265 [04.11.2020].

Kornelius, Stefan (2010b): „Amerikas Befreiung“. Süddeutsche Zeitung.de 17.5.2020. sueddeutsche.de/politik/obama-wird-us-praesident-amerikas-befreiung-1.525736 [04.11.2020].

Kornelius, Stefan (2010c): „Obama für alle“. Süddeutsche Zeitung.de 17.5.2010. sueddeutsche.de/politik/barack-obama-in-berlin-obama-fuer-alle-1.575735 [04.11.2020].

Krauel, Torsten (2011): „Ich sage es doch. Es ist nur eine Frage, wie“. Welt.de 24.11.2011. welt.de/politik/article13733898/Ich-sage-es-doch-Es-ist-nur-eine-Frage-wie.html [04.11.2020]. 
Kruse, Birgit/Das Gupta, Oliver (2010): „Der deutsche - oder nur der bayerische Obama?“ Süddeutsche Zeitung.de 17.05.2020. sueddeutsche.de/bayern/csu-mit-guttenberg-der-deutsche-oder-nur-der-bayerische-obama-1.401178-0 [04.11.2020].

Kruse, Birgit/Jakat, Lena (2011): „Der fleißige Franke.“ Süddeutsche Zeitung.de 02.03.2011. sueddeutsche.de/politik/friedrich-neuer-innenminister-der-fleissige-franke-1.1066945 [04.11.2020].

Lehming, Malte (2012): „Redlichkeit und Vertrauen statt Attraktivität und Rebellentum“. Tagesspiegel.de 21.04.2012. tagesspiegel.de/meinung/politisches-personal-redlichkeit-undvertrauen-statt-attraktivitaet-und-rebellentum/6537058.html [04.11.2020].

Löwenstein, Stephan (2011): „Dick aufgetragene Demut“. Frankfurter Allgemeine Zeitung.de 23.02.2011. faz.net/aktuell/politik/die-guttenberg-affaere/guttenberg-im-bundestag-dickaufgetragene-demut-1596801.html [04.11.2020].

Markwardt, Nils (2015): „Merkels stilles Zaudern“. Zeit.de 19.07.2015. zeit.de/kultur/201507/angela-merkel-machtpolitik-zaudern-unterlassen?utm_referrer=https $\% 3 \mathrm{~A} \% 2 \mathrm{~F} \% 2 \mathrm{~F}$ [04.11.2020].

Mika, Bascha (2012): „Sind Langweiler die neuen Vorzeige-Politiker?“ Frankfurter Rundschau.de. fr.de/meinung/sind-langweiler-neuen-vorzeige-politiker-11314109.html [04.11.2020].

$\mathrm{NN}_{1}$ (2010a): „Merkel wehrt sich - Guttenberg springt bei“. Süddeutsche Zeitung.de 17.05.2010. sueddeutsche.de/politik/wahlkampfstil-der-cdu-merkel-wehrt-sich-guttenbergspringt-bei-1.170871 [04.11.2020].

$\mathrm{NN}_{2}$ (2010b): „Der Durchstarter“. Süddeutsche Zeitung.de 19.05.2010. sueddeutsche.de/politik/us-praesidentschaft-obama-kandidiert-der-durchstarter-1.914799 [04.11.2020].

$\mathrm{NN}_{3}$ (2013): „Gabriel gesteht Wahlkampf-Lügen“. Bild.de 28.6.2013. bild.de/politik/inland/maybrit-illner/gabriel-gesteht-wahlkampf-luegen-31026404.bild.html [04.11.2020].

$\mathrm{NN}_{4}$ (2016): „Habe lange gebraucht, meine Eitelkeit zu überwinden“. Welt.de 23.09.2016. welt.de/politik/deutschland/article158337714/Habe-lange-gebraucht-meine-Eitelkeit-zuueberwinden.html [04.11.2020].

$\mathrm{NN}_{5}$ (2017): „Eklat bei Maischberger: Bosbach verlässt verärgert die Sendung“. Merkur.de 13.07.2017. merkur.de/politik/zoff-wegen-jutta-ditfurth-eklat-bei-maischberger-bosbachverlaesst-veraergert-sendung-zr-8481743.html [04.11.2020].

Perger, Werner A. (2008): „Vorbild Down Under“. Zeit.de 28.02.2008. zeit.de/online/2008/10/sozialdemokraten-kolumne-perger/komplettansicht [04.11.2020].

Polke-Majewski, Karsten (2011): „Guttenbergs falsche Demut“. Zeit.de 24.02.2011. zeit.de/politik/deutschland/2011-02/guttenberg-plagiat-bundestag-kommentar [04.11.2020].

Prantl, Heribert (2010a): „Die Dampfmaschine“. Süddeutsche Zeitung.de 17.05.2010. sueddeutsche.de/politik/oskar-lafontaine-die-dampfmaschine-1.160786 [04.11.2020].

Prantl, Heribert (2010b): Die Frau vom Finanzamt“", Süddeutsche Zeitung.de 17.05.2010. sueddeutsche.de/politik/merkel-zum-konjunkturpaket-die-frau-vom-finanzamt-1.373821 [04.11.2020].

Sagatz, Kurt (2009): „Seid umworben, Millionen!“ Tagesspiegel.de 11.07.2009. tagesspiegel.de/gesellschaft/medien/obama-effekt-seid-umworben-millionen/1555038.html

[04.11.2020]. 
Schloemann, Johan (2010): „Wie war ich, Hannelore?“. Süddeutsche Zeitung.de 20.05.2010. sueddeutsche.de/politik/tv-duell-ruettgers-kraft-die-rhetorik-wie-war-ich-hannelore1.934364 [04.11.2020].

Wolff, Verena (2010): „Hat Obama einen Traum?“. Süddeutsche Zeitung.de 17.05.2010. sueddeutsche.de/politik/gleichberechtigung-schwarz-und-weiss-hat-obama-einen-traum1.696157 [04.11.2020]

\section{Verzeichnis der YouTube-Videos}

Baring, Arnulf (2011): „Der Schummelbaron - Frechheit siegt?“ Maischberger: YouTube 22.02.2011. youtube.com/watch? $\mathrm{v}=\mathrm{Ud} 756 \mathrm{ztcyBw}$ [04.11.2020].

Demmer, Ulrike (2011): „Der Schummelbaron - Frechheit siegt?“ Maischberger: YouTube 22.02.2011. youtube.com/watch? $\mathrm{v}=\mathrm{Ud} 756 \mathrm{ztcyBw}[04.11 .2020]$.

Ditfurth, Jutta (2011): „Der Schummelbaron - Frechheit siegt?“ Maischberger: YouTube 22.02.2011. youtube.com/watch? $\mathrm{v}=\mathrm{Ud} 756 \mathrm{ztcyBw}[04.11 .2020]$.

Guttenberg, Karl-Theodor zu (2009): „Guttenberg über Charisma und Authentizität“ 30.07.2009. mein vzwahlzentrale, youtube.com/watch? $\mathrm{v}=\mathrm{gl}$ aDqByjBQ [04.11.2020].

Guttenberg, Karl-Theodor zu (2011): „Karl-Theodor zu Guttenberg (CSU), 21.2.2011 Stellungnahme $\quad \mathrm{zu} \quad$ Plagiatsvorwürfen“, $\quad$ YouTube 22.02 .2011$. youtube.com/watch?v=f8kfKXfUj9M [04.11.2020].

Lorenz, Antje (2018): „Emotionale Überraschung am Ende der Amtszeit. Wie Barack Obama Joe Biden zum Weinen bringt“. Welt Netzreporterin Antje Lorenz, 12.1.2018. youtube.com/watch? $\mathrm{v}=-\mathrm{SXW} 996 \mathrm{MgiA} \&$ feature=youtube; [04.11.2020].

„Rudi Völler rastet aus“: YouTube 11.08.2017. youtube.com/watch?v=-vck9JFYc88 [04.11.2020]. 